\title{
Statistische Theorie der Phasenumwandlung von Paraffinkristallen*
}

\author{
Von Friedhelm Eicker ** \\ Aus dem Institut für Theoretische Physik an der Universität Mainz \\ (Z. Naturforschg. 13 a, 126-143 [1958] ; eingegangen am 4. November 1957)
}

\begin{abstract}
Unter der Voraussetzung starrer Moleküle wird mit einem van der WaAssschen Ansatz ein Ausdruck für das Wechselwirkungspotential zweier drehbarer Nachbarmoleküle im Paraffinkristall in Gestalt einer Fourier-Entwicklung hergeleitet, wobei die Zickzackstruktur der C-Kette vernachlässigt ist. Damit läßt sich die freie Energie für die Grundfigur des Kristalls in der monoklinen und in der hexagonalen Phase aufstellen; Gleichsetzen beider ergibt die Umwandlungstemperatur. Die Nahordnung der Nachbarn eines Moleküls wird durch Verteilungsfunktionen für die Konfigurationen der einfachsten Kristallstrukturelemente berücksichtigt (BETHE-Approximation) ; ein Fernordnungsparameter tritt nicht auf. Die Entropie wird ähnlich wie beim Ising-Modell nach der von KIKUCHI allgemein dargestellten Methode kombinatorisch bestimmt; im Anhang sind dazu einige andere Möglichkeiten der Berechnung angegeben. Die freie Energie wird unter Heranziehung geeigneter Rirzscher Ansätze - im Anhang auch direkt - und quadratischer Näherungen für die Entropieintegrale variiert, wobei allgemeine Symmetrierelationen für die Verteilungsfunktionen und als Parameter unter anderem auch die Achsabstände der Molekülpaare eingehen. Der mittlere Winkel der Moleküle, Achsabstände, Umwandlungstemperatur sowie die Umwandlungswärme ergeben sich größenordnungsmäßig richtig; die Umwandlung ist von erster Ordnung. Eine Kettenlängenabhängigkeit, die den experimentellen Befunden nahekommt, erhält man allerdings erst, wenn die Voraussetzung starrer Moleküle fallen gelassen wird; die Abhängigkeit wird verbessert mit Hilfe einer einfachen, klassisch-statistischen Theorie der Verdrillung, die auf Überlegungen von Szigeti basiert. Die zum Teil noch ungenauen Resultate lassen sich durch Verwendung besserer Näherungen verschärfen, wobei der mathematische Aufwand allerdings erheblich wächst. Es ergibt sich aus der Theorie nicht, in welchem Kettenlängenbereich die Phasenumwandlung auftritt.

Im Anhang wird auf das Rotationspotential eines Moleküls in Abhängigkeit vom Drehwinkel bei festen Nachbarn in Weiterführung MüLlenscher Rechnungen sowie auf einige Bemerkungen zur Hoffmanschen Theorie der Paraffinumwandlung und zur Quantenmechanik behinderter Rotatoren eingegangen.
\end{abstract}

\section{Problemstellung}

Struktur und thermische Eigenschaften der Paraffinkristalle sind seit langem weitgehend bekannt ${ }^{1}$. Die Erscheinung, welche Gegenstand dieser Arbeit ist, ist die Umwandlung der Kristalle von der monoklinen in die hexagonale Phase mit zunehmender Temperatur, die bei Kettenlängen von 18-36 CAtomen pro Paraffinmolekül und Temperaturen bis zu $15{ }^{\circ} \mathrm{C}$ unter dem Schmelzpunkt auftritt. Das Problem ist hauptsächlich die Bestimmung der Um. wandlungstemperatur $T_{\mathrm{u}}$.

Es handelt sich um einen Übergang erster Ordnung, er besitzt eine Wärmetönung. Qualitativ wird dieser Übergang als Wechsel der vorherrschenden Bewegungsform der Moleküle von Schwingungen um jeweils eine von zwei bestimmten Richtungen im monoklinen Zustand zu freier Rotation im hexagonalen Zustand gedeutet. Davon ausgehend hat man häufig die Moleküle als behin-

* Diss. Universität Mainz 1956.

* Jetzt: Mathematisches Institut der Universität Mainz.

1 vgl. etwa den zusammenfassenden Bericht von V. DANiel. Adv. Phys. 2, 450 [1953]. derte, starre, bandförmige Rotatoren in einem mittleren, durch die Nachbarn bestimmten Potentialfeld idealisiert. Aus der freien Energie läßt sich dann bei Hinzunahme einiger zusätzlicher Forderungen $T_{\mathrm{u}}$ bestimmen. Der Bewegungszustand der Nachbarn geht nicht ein, die Nahordnung bleibt unberücksichtigt. Der Ordnungszustand des Kristalls wird vielmehr durch einen BRAGG-WILliamschen Fernordnungsparameter in Rechnung gestellt, bei dessen Definition sich aber die charakteristischen Schwierigkeiten dieser Theorie ergeben, auf die bereits Fowler $^{2}$ hingewiesen hat und die von KIRKwood ${ }^{3}$ durch Einführung von Verteilungsfunktionen für die Konfigurationen der Partikel z. Tl. umgangen wurden. Seitdem hat diese Theorie viele Weiterentwicklungen und Anwendungen vor allem auf das Ising-Modell des Ferromagneten ${ }^{4}$ erfahren. Eine Anwendung auf den Paraffinkristall hat u. a. HoffMAN ${ }^{5}$ unter Annahme eines stark schematisierten Potentialverlaufs gegeben. Eine genauere Bestim-

2 R. H. Fowler, Proc. Roy. Soc., Lond. A 149, 1 [1935].

3 J. G. Kirkwood, J. Chem. Phys. 8, 205 [1940].

4 T. Nakamura, J. Phys.-Math. Soc. Japan 7, 264 [1952].

5 J. D. Hofrman, J. Chem. Phys. 20, 541 [1952]. 
mung des dabei benötigten Potentialverlaufs ist in dieser Arbeit versucht worden, vgl. Anhang 6.

Eine grundsätzlich andere Theorie, welche aus der BETHE-Approximation des IsING-Modells entwickelt worden ist, benutzt außer einfachen Struktureigenschaften nur das einfacher zu bestimmende Wechselwirkungspotential zweier Moleküle. Die Nahordnung wird in vollem Umfang berücksichtigt, ein Fernordnungsparameter tritt daher nicht auf. Diese Theorie, welcher übrigens die erste weitgehend angeglichen werden kann, wird in 3. und 4. zugrunde gelegt. Anwendungen sind bisher auf das diskontinuierliche ${ }^{6}$ und das kontinuierliche ${ }^{7}$ Ising-Modell sowie z. B. auf ein diskontinuierliches Modell des Paraffins ${ }^{8,9}$ gemacht worden. Im letzteren werden drei fixe Stellungen postuliert, welche jedes Molekül annehmen darf.

Es zeigt sich bei allen Rechnungen, daß die Umwandlungstemperatur $T_{\mathrm{u}}$ des Paraffinkristalls der Kettenlänge $n$ proportional sein müßte, wenn man die Moleküle als starr ansieht. Die Experimente ${ }^{10}$ ergeben dagegen angenähert

$$
T_{\mathrm{u}} \approx 268,68+2,1917 n\left[{ }^{\circ} \mathrm{K}\right] \quad\left( \pm 0,03^{\circ}\right)
$$

für gerade $n=18 \ldots 36$, für ungerade geringfügige Abweichungen. Diese schwache Abhängigkeit läßt sich in der Theorie nur durch Berücksichtigung der Verdrillung annähern, vgl. etwa Hosoya ${ }^{8}$ und $\mathrm{K}_{\mathrm{A}}$ KIUCHI ${ }^{9}$. SzIGETI ${ }^{11}$ zieht zur Erklärung verwandter dielektrischer Effekte die Boltzmann-Statistik heran; ein ähnlicher Weg wird in $\mathbf{5}$. beschritten.

\section{Das Modell und sein Wechselwirkungspotential}

Die Struktur des Paraffinkristalls ${ }^{1}$ ist hauptsächlich von MüLLER ${ }^{12}$ untersucht worden; die hier wesentlichen Daten sind in Abb. 1 wiedergegeben. Außer in 5. wird die C-Kette stets als starr, gestreckt und nicht verdrillt angesehen. Die Moleküle bilden Schichten. Ihre Achsen stehen senkrecht zur Schichtebene. Die Durchstoßpunkte der Achsen bilden in der Schichtebene ein rechtwinkliges Gitternetz mit den Gitterkonstanten $a$ und $b$ (Abb. 1). Die Molekülebenen bilden mit der Richtung der Seite $a$ nach MüLLER mittlere Winkel von etwa $\pm 30^{\circ}$, nach

6 z. B. R. Kiкuchi, Phys. Rev. 81, 988 [1951].

7 T. OGuchi u. Y. Takagi, J. Phys.-Math. Soc. Japan 7, 145 [1952].

8 S. Hosoya, J. Phys.-Math. Soc. Japan 9, 524 [1954].

9 Y. Kakiuchi, J. Phys.-Math. Soc. Japan 6, 313 [1951]. neueren Messungen Bunss ${ }^{13}$ an sehr langen Molekülen $\pm 48,8^{\circ}$. Die Zentren der van der WaAlsschen Kräfte und die Massen der $\mathrm{CH}_{2}$-Gruppen sollen auf zwei Parallelen zur Molekülachse im Abstand der Ladungsschwerpunkte $r=0,6 \AA$ gleichmäßig verteilt sein; die Parallelen bilden mit der Achse die Molekülebene, die als Ganzes drehbar ist.

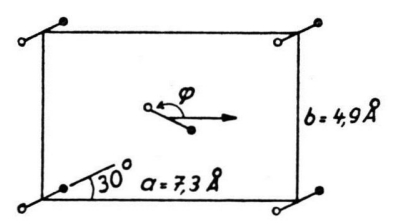

Abb. 1. Projektion der Molekülebenen einer Grundfigur auf die Schichtebene. Offene Kreise bedeuten C-Atome in einer Ebene. Nach MüLLER.

Die folgende zweidimensionale Theorie - die Wechselwirkung von Molekülen verschiedener Schichten aufeinander wird vernachlässigt - kann daher keine Effekte liefern, die auf der Zickzackstruktur oder auf der Geradheit bzw. Ungeradheit von $n$ beruhen.

Die Molekülebenen eines beliebig herausgegriffenen, benachbarten Paares mögen mit der Ebene durch die beiden Achsen die Winkel $\psi$ und $\chi$ bilden (s. Abb. 2). Das zu berechnende Potential zwischen

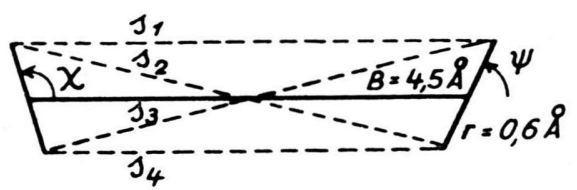

Abb. 2. Die ein Molekülpaar kennzeichnenden Größen $B, r$, $\psi, \chi, s_{1}, \ldots, s_{4}$.

beiden Molekülen ist in beiden Winkeln $\pi$-periodisch. Der Abstand $B$ der beiden Achsen beträgt etwa 4,5 $\AA$. Zwischen zwei Äthylengruppen verschiedener Moleküle möge ein Potential der Form

$$
u=-\frac{\mu}{R^{6}}+P e^{-R / \varrho}
$$

gelten. $R$ bedeutet den Abstand zwischen den Schwerpunkten der positiven Ladung, $\mu, P$, $\varrho$ sind Kon-

10 J.D. Hoffman u. B. F. Decker, J. Phys. Chem. 57, 520 [1953].

11 B. Szigeti, Trans. Faraday Soc. 48, 400 [1952]; Proc. Roy. Soc., Lond. B 65, 19 [1952].

12 A. Müller, Proc. Roy. Soc., Lond. A 120, 437 [1928].

13 C. W. Bunn, Trans. Faraday Soc. 35, 482 [1939]. 
stanten. Dieser Ansatz ist üblich bei kleineren Molekülen ${ }^{14}$. Wegen des edelgasähnlichen Charakters der Verteilung der Elektronendichte (vgl. etwa 1:3 oder ${ }^{1}$ ) in Teilen der äußeren Schalen ist ein Vergleich der Konstanten für die Äthylengruppe und für Neon oder Methan sinnvoll. Es ist für

Neon: ${ }^{14} \quad \mu=9 \cdot 10^{-12} \operatorname{erg} \AA^{-6}$ :

$$
\begin{aligned}
& P=2,57 \cdot 10^{-9} \mathrm{erg} ; \\
& \varrho=0,235 \AA,
\end{aligned}
$$

Methan: $\quad \mu=2,86 \cdot 10^{-10} \mathrm{erg} \AA^{-6}$;

$$
P=1,695 \cdot 10^{-8} \mathrm{erg} \text {; }
$$$$
\varrho=0,316 \AA \text {. }
$$

Bei Neon sind 10 positive Ladungseinheiten im Zentrum konzentriert, bei Methan 6; wir erwarten daher für die Äthylengruppe ein Volumen und demgemäß einen Gleichgewichtsabstand $R_{\min }$, welche zwischen den Werten für Neon und Methan liegen. Berücksichtigt man ferner die Sublimationswärme und den Gleichgewichtswinkel (vgl. Anh. 6.), so wird man etwa zu folgenden Zahlen für die Äthylengruppe geführt:

$$
\begin{aligned}
& \mu=1,5 \cdot 10^{-10} \mathrm{erg} \AA^{-6} ; \\
& P=3,6 \cdot 10^{-10} \mathrm{erg} ; \\
& \varrho=0,42 \AA .
\end{aligned}
$$

Genauere Werte sind der Literatur zur Zeit noch nicht zu entnehmen.

Das gesamte Potential einer Gruppe soll sich additiv aus den Energien (2,1) zwischen dieser Gruppe und sämtlichen anderen der Nachbarmoleküle zusammensetzen. Die Moleküle werden für die Berechnung als unendlich lang angenommen. Der Abstand zweier Linienelemente auf den Achsenparallelen sei

$$
R=\sqrt{s_{i}^{2}+\xi^{2}}
$$

(Abb. 3). Dann ist das Potential zwischen einer Gruppe und einer Parallelen, d. h. einer Reihe von Äthylengruppen eines anderen Moleküls,

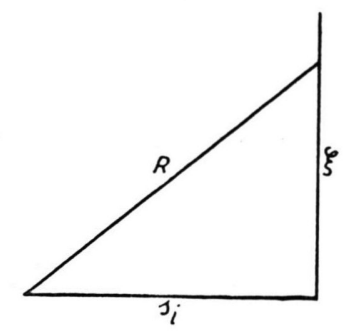

Abb. 3. Der Abstand $R$ zweier Linienelemente.

$$
u_{\mathrm{G}}=\int_{-\infty}^{+\infty}\left(-\frac{\mu}{R^{6}}+P e^{-R / \varrho}\right) \frac{\mathrm{d} \xi}{2,51},
$$

wo 2,51 $\AA$ der Abstand zweier Gruppen derselben Reihe ist und $R$ und $\xi$ in $\AA$ gemessen wird. Man erhält in guter Näherung

$$
u_{\mathrm{G}}=-\frac{0,47 \mu}{s_{i}^{5}}+\sqrt[V]{\varrho s_{i}} P e^{-s_{i} / \varrho} .
$$

Daraus folgt als mittleres Potential zwischen einer linienförmigen, auf die beiden Parallelen verteilt gedachten Gruppe und einem Molekül

$$
u_{\mathrm{G}, \mathrm{M}}=-\frac{0,47}{2} \mu \sum_{i=1}^{4} \frac{1}{s_{i}{ }^{5}}+V \varrho \frac{P}{2} \sum_{i=1}^{4} V s_{i} e^{-s_{i} / \varrho} .
$$

Mit der Näherung (vgl. Abb. 2)

$s_{1}=B+r(\cos \psi-\cos \chi), \quad s_{2}=B-r(\cos \psi-\cos \chi)$, $s_{3}=B+r(\cos \psi+\cos \chi), \quad s_{4}=B+r(\cos \chi-\cos \psi)$

folgen nach längerer Zwischenrechnung die Koeffizienten der in $\psi$ und $\chi$ symmetrischen FounierEntwicklung für das Potential zweier Moleküle der Länge $n$

$$
\begin{aligned}
U=(n-1)[ & a_{0}+a_{1}(\cos 2 \chi+\cos 2 \psi) \\
& +a_{2}(\cos 4 \chi+\cos 4 \psi) \\
& \left.+\ldots+a_{11} \cos 2 \chi \cos 2 \psi+\ldots\right],
\end{aligned}
$$

worin $n-1$ statt $n$ geschrieben ist, weil die Endgruppen nur etwa den halben Beitrag liefern. Es ist, wobei $z=r / B \approx 0,13$ und $I_{n}(x)$ eine modifizierte Besselsche Funktion bedeutet,

$$
\begin{gathered}
a_{0} \approx-{ }_{B^{5}}^{3.8 \prime \prime}\left(\frac{1}{4}+3,75 z^{2}+39,3 z^{4}+318 z^{5}\right) \\
+2 \sqrt{\varrho B} P e^{-B / \varrho} I_{0}{ }^{2}\left(\frac{r}{\varrho}\right) \\
\approx\left(-\frac{1,87}{B^{5}}+24,7 e^{-B / 0.42}\right) 10^{-10} \mathrm{erg}
\end{gathered}
$$

$$
\begin{aligned}
a_{1} \approx & -\frac{3,8 \mu}{B^{5}} z^{2}\left(1,88+35 z^{2}+369 z^{4}+3030 z^{6}\right. \\
& \left.+21500 z^{8}\right)+4 \sqrt{\varrho B} P \mathrm{e}^{-B / \varrho} I_{0}(r / \varrho) I_{2}(r / \varrho) \\
\approx & \left(-\frac{5.35}{B^{7}}+9,5 e^{-B / 0,42}\right) 10^{-10} \mathrm{erg},
\end{aligned}
$$$$
a_{11} \approx-\frac{3,8 \mu}{B^{5}} z^{4}\left(26,2+394 z^{2}+3790 z^{4}+25000 z^{6}\right)
$$$$
+8 \sqrt{\varrho B P} P e^{-B / \varrho} I_{2}^{2}(r / \varrho)
$$$$
\approx\left(\frac{25,5}{B^{9}}+3,67 e^{-B / 0,42}\right) 10^{-10} \mathrm{erg} .
$$

14 etwa H. A. Stuart, Struktur des freien Moleküls, SpringerVerlag, Berlin 1952, S. $40 \mathrm{ff}$. 
In den Endformeln ist $B$ in $A$ zu messen; man erhält sie durch Vernachlässigung der langsam mit $B$ veränderlichen Funktionen gegenüber den schnell veränderlichen bei mittleren $B$-Werten von etwa $4,5 \AA$ (Abb. 4). Es sei angemerkt, daß sich die Ausdrücke $(2,4)$ bis $(2,6)$ für die zu Methan gehörigen Konstanten $\mu, P, \varrho$ von den angegebenen wenig unterscheiden.

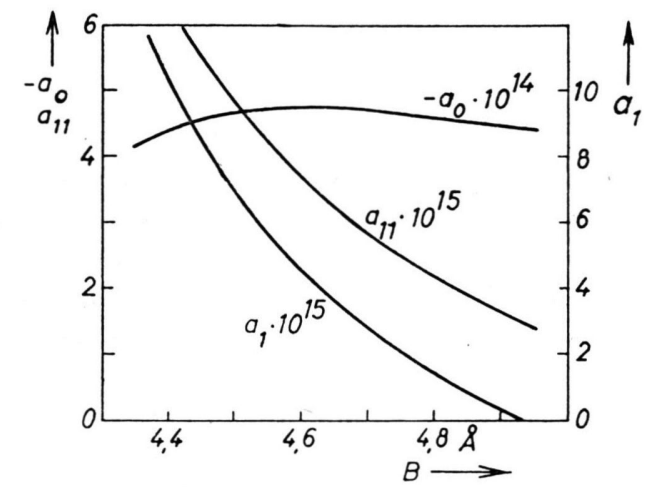

Abb. 4. Die Fourier-Kocffizienten des Wechselwirkungspotentials als Funktionen von $B$.

Wir legen nun It. Abb. 5 im Kristall eine Richtung $p=0$ fest, gegen die hinfort alle Winkel gemessen werden (Richtung der Seite $a$ ). Bildet die Ebene eines Paares mit dieser Richtung den Winkel $\alpha$, die Moleküle die Winkel $\varphi$ und $\psi$ gegen die Ebene des Paares, so ist in $(2,3) \chi$ durch $\varphi-\alpha$ und $\psi$ durch $\psi-\alpha$ zu ersetzen. Man erhält so für die Paare 1,

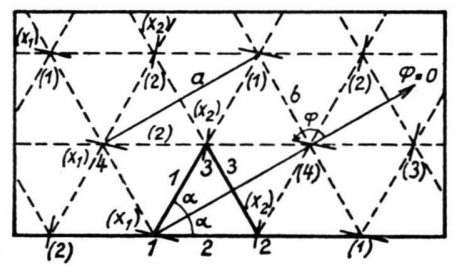

Abb. 5. Zur Klassifizierung und Orientierung der Moleküle und Molekülpaare in einer Schicht. Die kurzen Striche bedeuten Moleküle.

2,3 (Abb. 5) die in $\varphi$ und $\psi$ symmetrischen Wechselwirkungspotentiale $U_{i}(\varphi, \psi), i=1,2,3$; darin ist $a_{k, l}^{(i)} \equiv a_{k l}\left(B_{i}\right)$ für $i=\grave{i}$ und 3 gesetzt. Es gilt fü! die Abstände $B$ der Paare 1 und $2 B_{1}=B_{2}$. Damit erhält man schließlich für die Wechselwirkungspotentiale zwischen den zwei Molekülen der Paare 1, 2, 3 (Abb. 5)

$$
\begin{aligned}
& U_{1}(\varphi, \psi)=(n-1)\left\{a_{0}^{(1)}+a_{1}^{(1)}[\cos 2(\varphi-\alpha)\right. \\
& \left.+\cos 2(\psi-\alpha)]+a_{11}^{(1)} \cos 2(\varphi-\alpha) \cos 2(\psi-\alpha)\right\}, \\
& U_{2}(\varphi, \psi)=(n-1)\left\{a_{0}^{(1)}+a_{1}^{(1)}[\cos 2(\varphi+\alpha)\right. \\
& \left.\quad+\cos 2(\psi+\alpha)]+a_{11}^{(1)} \cos 2(\varphi+\alpha) \cos 2(\psi+\alpha)\right\}, \\
& U_{3}(\varphi, \psi)=(n-1)\left\{a_{0}^{(3)}+a_{1}^{(3)}\left[\cos 2\left(\varphi+\frac{\pi}{2}\right) \quad(2,7)\right.\right. \\
& \left.\left.+\cos 2\left(\psi+\frac{\pi}{2}\right)\right]+a_{11}^{(3)} \cos 2\left(\varphi+\frac{\pi}{2}\right) \cos 2\left(\psi+\frac{\pi}{2}\right)\right\} \\
& =(n-1)\left[a_{0}^{(3)}-a_{1}^{(3)}(\cos 2 \varphi+\cos 2 \psi)\right. \\
& \left.\quad+a_{11}^{(3)} \cos 2 \varphi \cos 2 \psi\right] .
\end{aligned}
$$

Es sei noch angemerkt, daß in der Summe der Wechselwirkungsenergien eines Moleküls vom Winkel $\varphi$ und aller seiner sechs Nachbarn im hexagonalen Fall $(\alpha=\pi / 6)$ alle linearen, nicht mit $\pi / 3$ periodischen Fourier-Komponenten, die von $\varphi$ abhängen, verschwinden wegen

$$
\cos x+\cos \left(x+\frac{2 \pi}{3}\right)+\cos \left(x-\frac{2 \pi}{3}\right)=0 .
$$

\section{Verteilungsfunktionen und die konfigurative freie Energie}

Die Aufgabe ist, die Umwandlungstemperatur $T_{\text {" }}$ statistisch zu berechnen. Dazu bestimmen wir die freie Energie

$$
F=E-T S
$$

für die monokline und die hexagonale Phase in der Nähe der Umwandlungstemperatur und setzen dann beide gleich. Man kann sich dabei auf den konfigurativen oder potentiellen Anteil der freien Energie beschränken, da der kinetische Anteil von der Kristallform unabhängig ist und sich also aus der Gleichung forthebt; dies geht unmittelbar aus dem GibBsschen Zustandsintegral hervor. $E$ und $S$ bedeuten hinfort nur den konfigurativen Anteil von Energie und Entropie. Wir berechnen beide getrennt für eine Grundfigur ${ }^{6}$ (basic figure) im Kristall, als welche wir den Rhombus 1, 2, 3, 4 (Abb. 5) wäh. len. Es sind nunmehr Verteilungsfunktionen (Wahrscheinlichkeiten) für die Konfigurationen verschiedener Strukturelemente einzuführen ${ }^{6,7}$. Als Strukturelemente unterscheiden wir:

a) vier Arten (sublattices) von Molekülen, näm. lich $1 ; 2 ; 3 ; 4$ oder (1); (2); (3); (4).

b) drei verschiedene Arten von Molekülpaaren $1 ; 2 ; 3$. 
c) zwei Arten Dreiecke: 1, 2, 3 und 1, 3, 4 .

d) den Rhombus 1, 2, 3, 4 .

Die zu a), b), c) gehörigen Verteilungsfunktionen werden folgendermaßen erklärt: $x_{1}(\varphi) \Delta \varphi$ gibt die Wahrscheinlichkeit dafür an, daß der Winkel des Moleküls 1, 4, (1) oder (4) im Intervall $\Delta \varphi$ um den Winkel $\varphi$ liegt. $x_{2}(\varphi)$ gibt entsprechend die Richtungsverteilung von Molekülen 2, 3, (2), (3) an. $p_{1}\left(\varphi_{1}, \varphi_{3}\right)$ gibt den Bruchteil der Paare 1 an, bei denen das Molekül 1 den Winkel $\varphi_{1}$, das Molekül 3 den Winkel $\varphi_{3}$ hat. Analog ist $p_{2}\left(\varphi_{1}, \varphi_{2}\right)$ und $p_{3}\left(\varphi_{2}, \varphi_{3}\right)$ sowie $w\left(\varphi_{1}, \varphi_{2}, \varphi_{3}\right)$ für das Dreieck $1,2,3$ erklärt. Es gelten definitionsgemäß die Relationen und Normierungen

$$
\begin{aligned}
x_{1}\left(\varphi_{1}\right)= & \int_{0}^{2 \pi} p_{1}\left(\varphi_{1}, \varphi_{3}\right) \mathrm{d} \varphi_{3}=\int_{0}^{2 \pi} p_{2}\left(\varphi_{1}, \varphi_{2}\right) \mathrm{d} \varphi_{2}, \\
x_{2}\left(\varphi_{2}\right)= & \int_{0}^{2 \pi} p_{1}\left(\varphi_{1}, \varphi_{2}\right) \mathrm{d} \varphi_{1}=\int_{0}^{2 \pi} p_{2}\left(\varphi_{1}, \varphi_{2}\right) \mathrm{d} \varphi_{1} \\
= & \int_{0}^{2 \pi} p_{3}\left(\varphi_{2}, \varphi_{3}\right) \mathrm{d} \varphi_{3}=\int_{0}^{2 \pi} p_{3}\left(\varphi_{3}, \varphi_{2}\right) \mathrm{d} \varphi_{3}, \\
& \int_{0}^{2 \pi} x_{1}(\varphi) \mathrm{d} \varphi=\int_{0}^{2 \pi} x_{2}(\varphi) \mathrm{d} \varphi=1, \\
p_{1}\left(\varphi_{1}, \varphi_{3}\right)= & \int_{0}^{2 \pi} w\left(\varphi_{1}, \varphi_{2}, \varphi_{3}\right) \mathrm{d} \varphi_{2}, \\
p_{2}\left(\varphi_{1}, \varphi_{2}\right)= & \int_{0}^{2 \pi} w\left(\varphi_{1}, \varphi_{2}, \varphi_{3}\right) \mathrm{d} \varphi_{3}, \\
p_{3}\left(\varphi_{2}, \varphi_{3}\right)= & \int_{0}^{2 \pi} w\left(\varphi_{1}, \varphi_{2}, \varphi_{3}\right) \mathrm{d} \varphi_{1} .
\end{aligned}
$$

Als mittlere Energie pro Molekül erhält man damit

$$
\begin{array}{r}
E=\int_{0}^{2 \pi}\left\{U_{1}(\varphi, \psi) p_{1}(\varphi, \psi)+U_{2}(\varphi, \psi) p_{2}(\varphi, \psi)\right. \\
\left.+U_{3}(\varphi, \psi) p_{3}(\varphi, \psi)\right\} \mathrm{d} \varphi \mathrm{d} \psi, \quad(3,2) \\
U_{i}(\varphi, \psi) \text { nach }(2,7) .
\end{array}
$$

Zur Herleitung eines Ausdruckes für die Konfigurationsentropie bedienen wir uns einer Methode, welche in einer leicht zu verallgemeinernden Weise z. B. von $\mathrm{K}_{\text {IKUCHI }}{ }^{6}$ dargestellt worden ist; wir übernehmen z. Tl. die dort verwandten Bezeichnungen. Man denke sich das Gitter durch sukzessives Anfügen einzelner Moleküle, etwa 4, 3, (4), (3) usw., aufgebaut (Abb. 5). Alle schon vorhandenen Mole- küle und -paare mögen sich in der richtigen, durch $x_{i}, p_{i}, w$ gegebenen Verteilung befinden. Das Anfügen eines Moleküls, etwa 3, soll so geschehen, daß sich alle neu entstehenden Strukturelemente ebenfalls in der richtigen Verteilung befinden. Gefragt ist nun, wieviel verschiedene Anordnungen $G_{\mathrm{L}}$ es für die voneinander verschiedenen Konfigurationen in einer $L$ Serie gibt; $L$ ist dabei eine beliebig große ganze Zahl und spielt nur die Rolle einer Hilfsgröße, um von den Wahrscheinlichkeiten zu Häufigkeiten übergehen zu können. In der $L$-Serie komme jede Konfiguration genau entsprechend ihrer Wahrscheinlichkeit vor. Außerdem ist zur Unterscheidung einer endlichen Zahl von Konfigurationen der stetig durchlaufene Winkelbereich der Moleküle vorübergehend in endlich viele Stufen aufgeteilt; in der $L$-Serie gibt es dann z. B. $x_{2}\left(\varphi_{*}\right) L \Delta \varphi \equiv x_{2, x} L$ Moleküle 3 im $\Delta \varphi$-Intervall um $\varphi_{\varkappa}$. - Wir lassen zunächst die Paare 1 und 3 außer acht.

Nun kommt $x_{1, \lambda} L$-mal in der $L$-Serie beim Molekül 4 der Winkel $\varphi_{\lambda}$ vor; also gibt es

$$
\left(x_{1, \lambda} L\right) / \prod_{\varkappa}\left(p_{2, \lambda, \varkappa} L\right) !
$$

verschiedene Anordnungen von durch $\varkappa$ unterschiedenen Konfigurationen des Paares (2), bei denen das Molekül 4 den Winkel $\varphi_{\lambda}$ hat. Durchläuft auch 2 . alle seine Werte, so gibt es

$$
G=\left(X_{1}\right)_{L} /\left(P_{2}\right)_{L}
$$

verschiedene Anordnungen von unterschiedlichen Konfigurationen in der $L$-Serie, wobei

$\left(X_{i}\right)_{L}=\prod_{\lambda}\left(x_{i, \lambda} L\right) !, \quad\left(P_{i}\right)_{L}=\prod_{\lambda, x}\left(p_{i, \lambda, x} L\right) !$

bedeutet. Die Konfigurationen der Paare 1 und 3 werden durch je einen Korrekturfaktor $\Gamma_{1}$ und $\Gamma_{3}$ berücksichtigt; $\Gamma_{i}$ gibt die Wahrscheinlichkeit dafür an, daß das Paar $i$ in der richtigen Verteilung vorkommt. Nimmt man näherungsweise an, die Konfiguration des Moleküls 3 sei nach dem Anfügen des Paares (2) unabhängig von der Konfiguration des Moleküls 1 , so gäbe es $L ! /\left(X_{2}\right)_{L}$ Anordnungen für 3 in der $L$-Serie, und es wird

$$
\Gamma_{\mathbf{1}}=\frac{\left(X_{1}\right)_{L}}{\left(P_{1}\right)_{L}} / \frac{L !}{\left(X_{2}\right)_{L}}, \quad \Gamma_{\mathbf{3}}=\frac{\left(X_{2}\right)_{L}}{\left(P_{3}\right)_{L}} / \frac{L !}{\left(X_{2}\right)_{L}},
$$

wenn man bei $\Gamma_{3}$ die Konfiguration von Molekül 3 auch als unabhängig von 2 ansieht.

Damit wird die approximative Gesamtzahl der Anordnungen in der $L$-Serie 


$$
G_{L}=G \Gamma_{1} \Gamma_{3}=\frac{\left(X_{1}\right)_{L}^{2}\left(X_{2}\right)_{L}^{3}}{\left(P_{1}\right)_{L}\left(P_{2}\right)_{L}\left(P_{3}\right)_{L}(L !)^{2}} .
$$

Je größer diese Zahl ist, desto mehr Möglichkeiten gibt es, erlaubte Zustände zu realisieren; es handelt sich um eine thermodynamische Wahrscheinlichkeit, und die Boltzmannsche Formel ergibt daraus bis auf eine unwesentliche additive Konstante die Entropie. Unter Verwendung der Stirlingschen Formel folgt für kontinuierlichen Winkel $\varphi$ als Entropie pro Molekül

$$
\begin{array}{r}
\frac{S}{k}=\frac{1}{L} \ln G_{L}=2 \int_{0}^{2 \pi} x_{1}(\varphi) \ln x_{1}(\varphi) \mathrm{d} \varphi+\int_{0}^{2 \pi} x_{2}(\varphi) \ln x_{2}(\varphi) \mathrm{d} \varphi-\int_{0}^{2 \pi} \int_{0}\left\{p_{1}(\varphi, \psi) \ln p_{1}(\varphi, \psi)\right. \\
\left.+p_{2}(\varphi, \psi) \ln p_{2}(\varphi, \psi)+p_{3}(\varphi, \psi) \ln p_{3}(\varphi, \psi)\right\} \mathrm{d} \varphi \mathrm{d} \psi ;
\end{array}
$$

(vgl. auch Anhang 10.) ; $k=$ Boltzmann-Konstante.

Für das nächste angefügte Molekül erhält man zunächst einen anderen Entropieausdruck, doch wird er auf Grund später einzuführender Symmetrierelationen gleich $(3,7)$. - Mit $(3,2)$ und $(3,7)$ ist nunmehr ein Ausdruck für die freie Energie gewonnen.

\section{Minimalbedingungen für die freie Energie. Phasengleichgewicht}

In der Formel für die konfigurative freie Energie $F$ nach $(3,2)$ und $(3,7)$

$$
\frac{\boldsymbol{F}}{k T}=\frac{1}{k T} \int_{0}^{2 \pi} \int_{0}^{\pi}\left\{U_{1} p_{1}+U_{2} p_{2}+U_{3} p_{3}\right\} \mathrm{d} \varphi \mathrm{d} \psi-5 \int_{0}^{2 \pi} x \ln x \mathrm{~d} \varphi+\int_{0}^{2 \pi}\left\{p_{1} \ln p_{1}+p_{2} \ln p_{2}+p_{3} \ln p_{3}\right\} \mathrm{d} \varphi \mathrm{d} \psi
$$

(worin schon teilweise von den folgenden Nebenbedingungen Gebrauch gemacht ist) sind die Funktionen $p_{i}(\varphi, \psi), i=1,2,3$ zunächst noch unbekannt; sie sind unter Berücksichtigung aller Nebenbedingungen so zu bestimmen, daß $F$ minimal wird. Außer den Integralbeziehungen $(3,1)$ gelten für die monokline Phase folgende Nebenbedingungen:

$$
\begin{aligned}
& p_{1}\left(\varphi_{1}, \varphi_{3}\right)=p_{2}\left(-\varphi_{3},-\varphi_{1}\right), \\
& p_{3}\left(\varphi_{2}, \varphi_{3}\right)=p_{3}\left(\varphi_{3} \varphi_{2}\right), \\
& x_{1}\left(\varphi_{1}\right)=x_{2}\left(-\varphi_{1}\right) \equiv x\left(\varphi_{1}\right) .
\end{aligned}
$$

Jede auftretende Verteilungsfunktion ist in jedem Argument $\pi$-periodisch, weil die Zickzackstruktur außer acht gelassen ist. In dem Spezialfall des hexagonalen Gitters $\alpha=\pi / 6$ gilt außerdem noch

$$
p_{i}(\varphi, \psi)=p_{i}(\psi, \varphi), \quad i=1,2,3
$$

$$
\begin{aligned}
p_{1}\left(\varphi_{1}, \varphi_{3}\right)=p_{2}\left(\varphi_{1}-\frac{\pi}{3}, \varphi_{3}-\frac{\pi}{3}\right) & \\
& =p_{3}\left(\varphi_{1}+\frac{\pi}{3}, \varphi_{3}+\frac{\pi}{3}\right),
\end{aligned}
$$

$$
\begin{aligned}
& p_{3}\left(\varphi_{2}, \varphi_{3}\right)=p_{3}\left(-\varphi_{3},-\varphi_{2}\right) ; \\
& x_{1}(\varphi)=x_{2}(\varphi)=x_{1}\left(\varphi \pm \frac{\pi}{3}\right) ;
\end{aligned}
$$

eine Folgerung ist

$$
p_{1}(\varphi, \psi)=p_{1}\left(\frac{\pi}{3}-\varphi, \frac{\pi}{3}-\psi\right) .
$$

Die Richtigkeit dieser Nebenbedingungen erkennt man etwa durch Ausführung aller im jeweiligen Fall erlaubten Verschiebungen, Drehungen und Spiegelungen des Koordinatennetzes bei festgehaltenem Kristallgitter (vgl. Abb. 5). Mit dem Fourier-Ansatz

$$
p_{i}(\varphi, \psi)=\sum_{k, l} p_{k, l}^{(i)} e^{2 i(k \varphi+l \psi)}
$$

liefern die obigen Beziehungen im monoklinen Fall sofort folgende Gleichungen:

$$
x_{1}(\varphi)=2 \approx \sum_{k} p_{k, 0}^{(1)} e^{2 i k \varphi}=x_{2}(-\varphi),
$$

$$
p_{0}^{(i)}=\frac{1}{4 \pi^{2}}, \quad p_{k, 0}^{(1)}=p_{0,-k}^{(1)}=p_{0,-k}^{(3)}, \quad p_{k, l}^{(3)}=p_{l, k}^{(3)} .
$$

Die freie Energie im monoklinen Gitter wird mit $U_{i}(\varphi, \psi)$ nach $(2,7)$, wobei $B_{1}=B_{2}$ und $x_{1}(\varphi) \equiv x(\psi)$ gesetzt worden ist,

$$
\begin{gathered}
\frac{F}{k T}=\frac{1}{k T} \iint\left\{2 U_{1}(\varphi, \psi) p_{1}(\varphi, \psi)+U_{3}(\varphi, \psi) p_{3}(\varphi, \psi)\right\} \mathrm{d} \varphi \mathrm{d} \psi \\
\quad-5 \int x(\varphi) \ln x(\varphi) \mathrm{d} \varphi+\iint\left\{2 p_{1}(\varphi, \psi) \ln p_{1}(\varphi, \psi)+p_{3}(\varphi, \psi) \ln p_{3}(\varphi, \psi)\right\} \mathrm{d} \varphi \mathrm{d} \psi \\
=\tau\left\{2 a_{0}^{(1)}+a_{0}^{(3)}+4 \pi^{2}\left(p_{1,0}^{(1)}+p_{-1,0}^{(1)}\right)\left(a_{1}^{(1)} 2 \cos 2 \alpha-a_{1}^{(3)}\right)\right. \\
\left.+\pi^{2}\left[2 a_{1,1}^{(1)}\left(p_{1,1}^{(1)} e^{-4 i \alpha}+p_{-1,-1}^{(1)} e^{-4 i \alpha}+p_{1,-1}^{(1)}+p_{-1,1}^{(1)}\right)+a_{1,1}^{(3)}\left(p_{1,1}^{(3)}+2 p_{1,-1}^{(3)}+p_{-1,-1}^{(3)}\right)\right]\right\} \\
\quad-5 \int x(\varphi) \ln x(\varphi) \mathrm{d} \varphi+\iint\left\{2 p_{1}(\varphi, \psi) \ln p_{1}(\varphi, \psi)+p_{3}(\varphi, \psi) \ln p_{3}(\varphi, \psi)\right\} \mathrm{d} \varphi \mathrm{d} \psi
\end{gathered}
$$


mit

$$
\tau=(n-1) / k T .
$$

Speziell im hexagonalen Fall gilt ferner

$p_{k, l}^{(i)}=p_{l, k}^{(i)} ; \quad p_{k, l}^{(1)}=p_{-k,-l}^{(1)} e^{-2 i(k+l)(\pi / 3)} ; \quad p_{k, 0}^{(1)}=p_{k, 0}^{(3)}=p_{-k, 0}^{(1)}=p_{0, k}^{(1)} \equiv p_{k, 0} ; \quad p_{0,3 *+1}=p_{0,3 \varkappa+2}=0$.

Wegen $B_{1}=B_{2}=B_{3}$ setzen wir $B_{1} \equiv B, a_{k, l}^{(i)} \equiv a_{k, l}$ sowie $p_{k, l}^{(1)} \equiv p_{k, l} ; p_{1}(\varphi, \psi) \equiv p(\varphi, \psi)$; dann ist die hexagonale freie Energie

$$
\begin{gathered}
F_{\text {hex }} \\
k T
\end{gathered}=3 \tau\left\{a_{0}+2 \pi^{2} a_{1,1}\left(p_{1,-1}+e^{2 \pi i / 3} p_{1,1}\right)\right\}-5 \int x \ln x \mathrm{~d} \varphi+3 \iint p \ln p \mathrm{~d} \varphi \mathrm{d} \psi .
$$

Die freie Energie $(4,1)$ bzw. $(4,2)$ enthält nunmehx sämtliche Nebenbedingungen.

Wir rechnen zuerst den monoklinen Fall weiter. Für das Vorhandensein eines Minimums von $F$ nach $(4,1)$ ist es notwendig - jedoch keineswegs hinreichend - , daß sämtliche ersten Ableitungen nach den Veränderlichen $p_{k, l}^{(i)}, \alpha$ und $B_{1}$ verschwinden. Das liefert unter Weglassung der konjugiert komplexen Gleichungen zu $p_{-k,-l}^{(i)}=\overline{p_{k, l}^{(i)}}$ mit $\sin \alpha=B_{3} / 2 B_{1}$

$$
\begin{aligned}
& 0=4 \pi^{2} \tau\left(a_{1}^{(1)} 2 \cos 2 \alpha-a_{1}^{(3)}\right)-10 \pi \int e^{2 i \varphi} \ln x \mathrm{~d} \varphi \\
& +\iint\left\{2\left(e^{2 i \varphi}+e^{-2 i \psi}\right) \ln p_{1}+\left(e^{-2 i \varphi}+e^{-2 i \psi}\right) \ln p_{3}\right\} \mathrm{d} \varphi \mathrm{d} \psi, \\
& 0=10 \pi \int e^{2 i k \varphi} \ln x \mathrm{~d} \varphi+\iint\left\{2\left(e^{2 i k \varphi}+e^{-2 i k \psi}\right) \ln p_{1}+\left(e^{-2 i k \varphi}+e^{-2 i k \psi^{\prime}}\right) \ln p_{3}\right\} \mathrm{d} \varphi \mathrm{d} \psi \\
& \text { für }|k| \neq 1 \text {; } \\
& 0=\pi^{2} \tau a_{11}^{(1)} e^{4 i \alpha}+\iint e^{2 i(p+\psi)} \ln p_{1} \mathrm{~d} \varphi \mathrm{d} \psi, \\
& 0=\pi^{2} \tau a_{11}^{(1)}+\iint e^{2 i(\varphi-\psi)} \ln p_{1} \mathrm{~d} \varphi \mathrm{d} \psi, \\
& 0=\iint e^{2 i(k \varphi+l \psi)} \ln p_{1} \mathrm{~d} \varphi \mathrm{d} \psi=\iint e^{2 i(k \varphi+l \psi)} \ln p_{3} \mathrm{~d} \varphi \mathrm{d} \psi \\
& 0=\pi^{2} \tau a_{11}^{(3)}+\iint e^{2 i(\varphi+\psi)} \ln p_{3} \mathrm{~d} \varphi \mathrm{d} \psi, \\
& 0=\pi^{2} \tau a_{11}^{(3)}+\iint e^{2 i(\varphi-\psi)} \ln p_{3} \mathrm{~d} \varphi \mathrm{d} \psi, \\
& \text { für }|k l| \geqq 2 \text {; } \\
& 0=\frac{\mathrm{d} a_{0}^{(3)}}{\mathrm{d} B_{3}} B_{1} \cos \alpha-4 \pi^{2}\left(p_{1,0}^{(1)}+p_{-1,0}^{(1)}\right)\left(a_{1}^{(1)}-2-\sin 2 \alpha+\frac{\mathrm{d} a_{1}^{(3)}}{\mathrm{d} B_{3}} B_{1} \cos \alpha\right) \\
& +\pi^{2}\left[a_{11}^{(1)}\left(p_{1,1}^{(1)} e^{4 i \alpha}-p_{-1,-1}^{(1)} e^{-4 i \alpha}\right) 4 i+\frac{\mathrm{d} a_{1,1}^{(3)}}{\mathrm{d} B_{3}} B_{1} \cos \alpha\left(p_{1,1}^{(3)}+2 p_{1,-1}^{(3)}+p_{-1,-1}^{(3)}\right)\right], \\
& 0=\frac{\mathrm{d} a_{0}^{(1)}}{\mathrm{d} B_{1}}+\frac{\mathrm{d} a_{0}^{(3)}}{\mathrm{d} B_{3}} \sin \alpha+4 \pi^{2}\left(p_{1,0}^{(1)}+p_{-1,0}^{(1)}\right)\left(\frac{\mathrm{d} a_{1}^{(1)}}{\mathrm{d} B_{1}} \cos 2 \alpha-\frac{\mathrm{d} a_{1}^{(3)}}{\mathrm{d} B_{3}} \sin \alpha\right) \\
& +\pi^{2}\left[\frac{\mathrm{d} a_{1,1}^{(1)}}{\mathrm{d} B_{1}}\left(p_{1,1}^{(1)} e^{4 i \alpha}+p_{-1,-1}^{(1)} e^{-4 i \alpha}+p_{1,-1}^{(1)}+p_{-1,1}^{(1)}\right)+\frac{\mathrm{d} a_{1,1}^{(3)}}{\mathrm{d} B_{3}} \sin \alpha\left(p_{1,1}^{(3)}+2 p_{1,-1}^{(3)}+p_{-1,-1}^{(3)}\right)\right] .
\end{aligned}
$$

Es folgt damit aus $(4,1)$, daß die minimale freie Energie des monoklinen Gitters die Gestalt

$$
\begin{aligned}
F_{\min }=\tau & \left(2 a_{0}^{(1)}+a_{0}^{(3)}\right)-\frac{5}{2 \pi} \int \ln x \mathrm{~d} \varphi \\
& +\frac{1}{4 \pi^{2}} \iint\left\{2 \ln p_{1}+\ln p_{3}\right\} \mathrm{d} \varphi \mathrm{d} \psi
\end{aligned}
$$

hat. - Für die Funktionen $p_{(i)}(\varphi, \psi)$ dieser Variationsaufgabe lassen sich auch zwei Funktionalgleichungen aufstellen (vgl. Anh. 9.), von denen jedoch hier kein Gebrauch gemacht wird.

Die Gln. $(4,3)$ bis $(4,11)$ stellen ein kompliziertes implizites System für die Parameter $p_{k, l}^{(i)}, \alpha, B_{1}$ dar. Sind zu einem bestimmten Wert des Temperatur- parameters $\tau$ mehrere Lösungen vorhanden, so ist diejenige auszuwählen, für welche $\mathrm{Gl} .(4,12)$ den kleinsten Wert annimmt. Unter den Voraussetzungen, daß $F$ überhaupt ein absolutes Minimum sowie die nötigen Differenzierbarkeitseigenschaften besitzt, welche wir ungeprüft für das Weitere zugrunde legen, ist dann also jener kleinste Werte das abso. lute Minimum. Es zeigt sich, daß mathematisch den physikalischen Phasen verschiedene Typen von Lösungen des Variationsproblems entsprechen; jede Lösung ergibt einen Ast der mehrdeutigen Funktion $F(\tau)$. Die Schnittpunkte der Kurven zu jeweils kleinstem $F$ bestimmen die Umwandlungstemperaturen zwischen je zwei Phasen. 
Zur Vereinfachung des Systems $(4,3)$ bis $(4,11)$ entwickeln wir die Logarithmen bis zum quadratischen Glied

$$
\begin{aligned}
\ln x & \approx-\ln 2 \pi-1,5+4 \pi x-2 \pi^{2} x^{2}, \\
\ln p_{i} & \approx-\ln 4 \pi^{2}-1,5+8 \pi^{2} p_{i}-8 \pi^{4} p_{i}{ }^{2}
\end{aligned}
$$

und machen den Rirzschen Ansatz

$$
p_{k, 0}^{(1)}=0 \text { für }|k|>1, \quad p_{k, l}^{(i)}=0 \text { für }|k l|>1 .
$$

Wir setzen ferner

$$
p_{1,0}=r \cdot e^{i \delta}
$$

und erhalten durch Elimination aus den Gln. $(4,5)$ bis $(4,8)$ und $(4,12)$

$$
\begin{gathered}
p_{1,1}^{(1)}=-\frac{\tau}{16 \pi^{2}} a_{11}^{(1)} e^{-4 i \alpha}+4 \pi^{2} r^{2}, \\
p_{1,-1}^{(1)}=-\frac{\tau}{16 \pi^{2}} a_{11}^{(1)}+4 \pi^{2} r^{2} e^{2 i \delta}, \quad(4,14 ; \\
i_{1,1}^{(3)}=-\frac{\tau}{16 \pi^{2}} a_{11}^{(3)}+4 \pi^{2} r^{2} e^{-2 i \delta}, \\
p_{1-1}^{(3)}=-\frac{\tau}{16 \pi^{2}} a_{11}^{(3)}+4 \pi^{2} r^{2} ; \\
\frac{F_{\min }}{k T}=-\ln 2 \pi+\tau\left(2 a_{0}^{(1)}+a_{0}^{(3)}\right)-\frac{\tau^{2}}{8}\left(2\left(a_{11}^{(1)}\right)^{2}+\left(a_{11}^{(3)}\right)^{2}\right) \\
-16 r^{2} \pi^{1}\left\{96 r^{2} \pi^{4}-\cos 2 \delta \cdot \tau\left(a_{11}^{(1)}+\frac{a_{11}^{(3)}}{2}\right)\right. \\
\left.+1-\tau\left(a_{11}^{(1)} \cos 4 \alpha+\frac{a_{11}^{(3)}}{2}\right)\right\} .
\end{gathered}
$$

Gl. $(4,3)$ und die dazu konjugiert komplexe ergibt

$$
\begin{gathered}
0,0634-2(2 \pi r)^{2}=\underset{16 \pi^{2}}{\tau} a_{11}^{(1)} 2 \sin 4 \alpha \cot \delta,(4,16) \\
\cos \delta=\frac{\tau E}{2 \pi(2 \pi r)\left[474(2 \pi r)^{2}-1-\tau D\right]} \quad(4,17)
\end{gathered}
$$

mit den Abkürzungen

$E \equiv a_{1}^{(1)} 2 \cos 2 \alpha-a_{1}^{(3)}, \quad D \equiv a_{11}^{(3)}+a_{11}^{(1)}(1+\cos 4 \alpha)$.

Dieses Gleichungspaar hat in dem physikalisch interessierenden Wertebereich

$4,2<B_{1} \leqq B_{3}<5,5 ; 4 \cdot 10^{14} \lesssim \tau \lesssim 9 \cdot 10^{14}\left[\mathrm{erg}^{-1}\right]$

bis zu fünf verschiedene Lösungen mit $2 \pi r$ zwischen 0 und 0,18 . $\delta$ nimmt zu den der Größe nach geordneten $r$-Werten zunächst zwei dicht beieinanderliegende Werte wenig größer als $-\pi$, dann einen Wert bei etwa $+\pi / 4$ und schließlich je einen wenig kleiner als $\pi / 2$ bzw. wenig größer als $-\pi / 2$ an. $\mathrm{Zu}$ $E=0$ gibt es außerdem die Lösung $r=0$; das ist die Entartung zum hexagonalen Gitter. Mit Näherungen der einzelnen Lösungen $r, \delta$ geht man in die Gln. $(4,10 ; 4,11)$. Speziell für $2 \pi r \approx 0,178$, $\delta \approx \pm \pi / 2$ ergibt sich relativ leicht $\tau E$ als mittelbare Funktion von $B_{1}$ und $B_{3}$ durch punktweises Ausrechnen. Man findet durch einige Abschätzungen, daß nur diese und die Lösung $r=0$ für minimale Werte von $F$ in Frage kommen. Im fraglichen $\tau$-Bereich stellt sich eine geringfügige Abhängigkeit der $B_{i}$ von $\tau=(n-1) / k T$ heraus (Abb. 6). Danach nehmen beide Abstände zu bei zunehmender Temperatur oder bei abnehmender Kettenlänge. Während der erste Effekt experimentell bestätigt ist, finden sich für den zweiten, der natürlich modellbedingt sein kann, keine Angaben.

Für $r=0,178 / 2 \pi, \delta \approx \pm \pi / 2$ und $B_{1}, B_{3}$ laut Abb. 6 wird die freie Energie nach $(4,15)$

$$
\begin{aligned}
\frac{F_{\min }}{k T}=-\ln 2 \pi-10,8-\tau & 15,2 \cdot 10^{-14} \\
& -\tau^{2} 1,06 \cdot 10^{-29} .
\end{aligned}
$$

Dies ist die freie Energie der monoklinen Phase. Der mittlere Winkel der Moleküle ist $\delta / 2 \approx \pm \pi / 4$; Meßwerte liegen bei $30^{\circ}$ und $48,8^{\circ} 12,13$.

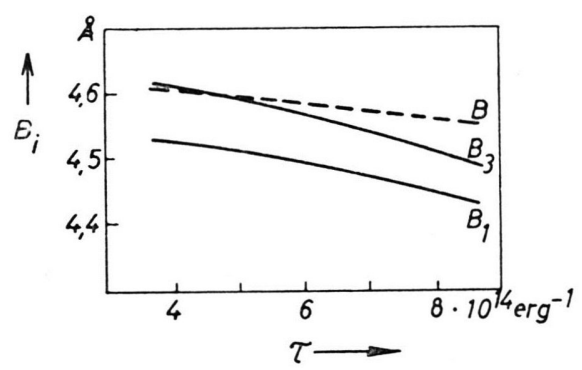

Abb. 6. Die Abstände $B_{i}$ als Funktionen von $\tau . B=$ Abstand im hexagonalen Gitter.

Für die Lösung $r=0$ verringert sich die Zahl der im oben benutzten Ritzschen Ansatz verfügbaren Parameter $p_{k, l}^{(i)}$ von fünf auf zwei; der zugehörige Näherungswert der freien Energie wird damit verhältnismäßig groß. Es empfiehlt sich deshalb folgender Ritzscher Ansatz mit vier Parametern:

$p_{k l} \equiv p_{k l}^{(1)}=0$ für $|k l|\left\{\begin{array}{l}=0, \text { außer } k=l=0 . \\ >2 .\end{array}\right.$

Wegen $E=0$ ist $B_{1}=B_{3} \equiv B$, es liegt ein hexagonales Gitter vor und es gelten die entsprechenden Symmetrierelationen. Man erhält aus

$$
\frac{\mathrm{d} a_{0}}{\mathrm{~d} B}=-\frac{\mathrm{d} a_{11}}{\mathrm{~d} B}
$$

den $\tau$-unabhängigen Wert $B=4,73 \AA$ und als freie Energie der hexagonalen Phase 


$$
\begin{aligned}
\frac{F_{\text {hex }}}{k T}=-\ln 2 \pi-18+3 \tau\left(a_{0}-a_{11}\right) \\
=-\ln 2 \pi-18-\tau 14,9 \cdot 10^{-14} .
\end{aligned}
$$

$r=0$ bedeutet, daß die Moleküle keine Richtung bevorzugen; sie „rotieren“ gleichmäßig.

Setzt man nun, um die Umwandlungstemperatur $T_{\mathrm{u}}$ zu erhalten, die beiden freien Energien $(4,19)$, $(4,21)$ gleich, so ergibt sich

$$
\tau_{\mathrm{u}} \approx 7 \cdot 10^{14} \mathrm{erg}^{-1}, \quad T_{\mathrm{u}} \approx \frac{n}{7 k} 10^{-14} \mathrm{erg} .
$$

Für $T<T_{\mathrm{u}}$ ist die freie Energie der monoklinen Phase kleiner als die der hexagonalen; der mono- kline Zustand ist dann also stabil. Für $T>T_{\text {u gilt }}$ das Umgekehrte, stabil ist der hexagonale Zustand.

Es handelt sich um einen Übergang erster Ordnung mit latenter Umwandlungswärme. Da nämlich die Volumänderung beim Phasenwechsel des festen Körpers vernachlässigt werden kann, gilt für die Umwandlungswärme

$$
\Delta Q \approx \Delta H \approx \Delta E=E_{\mathrm{hex}}-E_{\mathrm{mon}} .
$$

Darin ist $H$ die Enthalpie, $E_{\text {hex }}$ und $E_{\text {mon }}$ entnimmt man den Formeln $(4,1)$, $(4,2)$. Es gilt mit $(4,14)$ für $\tau=\tau_{\mathrm{u}}$

$$
\begin{aligned}
E_{\mathrm{mon}}= & (n-1)\left\{2 a_{0}^{(1)}+a_{0}^{(3)}+4 \pi^{2} r 2 \cos \delta\left(a_{1}^{(1)} 2 \cos 2 \alpha-a_{1}^{(3)}\right)+\pi^{2}\left[2 a _ { 1 1 } ^ { ( 1 ) } \left(-\frac{\tau}{4 \pi^{2}} a_{11}^{(1)}+4 \pi^{2} r^{2} 2(\cos 4 \alpha\right.\right.\right. \\
& \left.\left.+\cos 2 \delta))+a_{11}^{(3)}\left(-\frac{\tau}{4 \pi^{2}} a_{11}^{(3)}+4 \pi^{2} r^{2} 2(1+\cos 2 \delta)\right)\right]\right\}=-(n-1) \cdot 20 \cdot 10^{-14} \mathrm{erg} / \text { Molekül. }
\end{aligned}
$$

Ferner ergibt die Rechnung für den Ritzschen Ansatz $(4,20)$ :

$$
p_{1,1}=\frac{1}{4 \pi^{2}} e^{-2 \pi i / 3} ; \quad p_{1,-1}=\frac{1}{4 \pi^{2}},
$$

was in $(4,2)$ mit $B=4,73 \AA$

$$
\begin{aligned}
E_{\text {hex }} & =3(n-1)\left(a_{0}+a_{11}\right) \\
& =-(n-1) \cdot 13,4 \cdot 10^{-14} \mathrm{erg} / \text { Molekül }
\end{aligned}
$$

liefert. Damit wird die Umwandlungswärme für den Ubergang von der monoklinen zur hexagonalen Phase angenähert

$$
\Delta Q \approx 18 \ldots 36 \mathrm{kcal} / \mathrm{Mol} \text {, }
$$

je nach der Größe von $n$ zwischen 18 und 36. Die stark streuenden Meßwerte dieser Größe ${ }^{15}$ schwanken zwischen 3,6 und $11,5 \mathrm{kcal} / \mathrm{Mol}$; man erhält also durch die Rechnung etwa die richtige Größenordnung, jedoch ist die angenäherte Proportionalität mit $n$ unbefriedigend.

Der oben gefundene Wert $\tau_{\mathrm{u}}$ liefert zwar recht genau die Größenordnung der Umwandlungstemperatur, doch wird er schon stark durch die Rechengenauigkeit und in nicht übersehbarer Weise natürlich auch durch die verschiedenen Potential- und Näherungsansätze beeinträchtigt. Das gefundene Ergebnis ist daher nur von geringer Bedeutung. Daß die Modellannahmen z. Tl. erheblich den wirklichen Sachverhalt unberücksichtigt lassen, zeigt sich hier wie in allen ähnlichen Untersuchungen in der fal-

15 W. F. S Eyer, R. F. Patterson u. J. L. Keays, J. Amer. Chem. Soc. 66, 179 [1944]. schen Kettenlängenabhängigkeit der Umwandlungstemperatur. Bessere Resultate lassen sich bei Berücksichtigung der Verdrillung erzielen, vgl. 5.

Hosoya ${ }^{8}$ gibt ebenfalls eine Formel für $T_{\mathrm{u}}$ an, welche für ein dreistufiges Potential des Paraffinmoleküls abgeleitet ist. Sie kann jedoch wegen einer mit unserem Potentialansatz nicht genau genug berechenbaren kleinen Differenz nicht zu einem Vergleich herangezogen werden.

Offensichtlich lassen sich nach der hier angewandten Methode auch andere Klassen von Kristallen unter sinngemäßer Abänderung des Potential- und Entropieansatzes behandeln. Zur Prüfung der Genauigkeit des Verfahrens ist allerdings das Paraffinmolekül wegen der vielfältigen Modellannahmen schlecht geeignet.

\section{Die Änderung der Potentialformel und der Ergebnisse bei Berücksichtigung der Molekül- verdrillung}

Für eine erste, überschlägige Rechnung genügt es, sämtliche $\mathrm{CH}_{2^{-}}$und $\mathrm{CH}_{3}$-Gruppen des Moleküls durch $n$ auf Kreisbahnen um die Achse drehbare, gleichartige Massenpunkte zu schematisieren; jedem Massenpunkt ist ein Richtungspfeil zugeordnet, welcher den Drehwinkel angibt (Abb. 7). Wenn das Molekül nicht angeregt ist, zeigen alle Pfeile in dieselbe Richtung; sie liegen dann in der Ebene der C-Atome. Der Winkel $\Phi_{v}$ der $v$-ten Gruppe werde durch den zugehörigen Pfeil gegen eine feste Ebene 
durch die Achse gemessen. Dann ist die innere potentielle Energie ${ }^{11}$ eines Moleküls bei kleinen Verdrillungen $\left|\Phi_{v}-\Phi_{v-1}\right|$

$$
E_{\mathrm{pot}}\left(\Phi_{1}, \ldots, \Phi_{n}\right)=\frac{3 A}{4} \sum_{v=1}^{n}\left(\Phi_{v}-\Phi_{v-1}\right)^{2},
$$

$n=$ Anzahl der C-Atome; $A=9,89 \cdot 10^{-13} \mathrm{erg} / \mathrm{CH}_{2}$. Paar nach TAYLOR ${ }^{16}$, nach SzIGETI ${ }^{11} A=8,63 \cdot 10^{-13}$ erg/ $\mathrm{CH}_{2}$-Paar. $A$ ergibt sich aus den Kräften zweier $\mathrm{CH}_{2}$ - oder $\mathrm{CH}_{3}$-Gruppen gegeneinander bei Verdrehung um die Verbindungslinie. Um eine Vorstellung von der Größenordnung der Verdrillung zu haben,

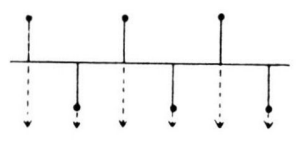

Abb. 7. Schema der Äthylengruppen als Massenpunkte mit Richtungspfeilen.

sei Szigetis Formel für die mittlere Differenz der Winkel zweier aufeinanderfolgender Gruppen und ihr Resultat für Zimmertemperatur angegeben:

$$
\left[\overline{\left(\Phi_{v}-\Phi_{v-1}\right)^{2}}\right]^{1 / 2}=\frac{\sin ^{2} \Theta / 2}{A} k T \approx 9^{\circ} ;
$$

darin ist $\Theta=109,5^{\circ}$ der (feste) Winkel zwischen drei C-Atomen.

Wir beschränken uns im folgenden auf die Anwendung der klassischen Statistik, doch ist damit zu rechnen, daß die Quantentheorie die Ergebnisse leicht modifiziert. Die Häufigkeit einer bestimmten Konfiguration des Moleküls ist dann gegeben durch

$$
w\left(\Phi_{1}, \ldots, \Phi_{n}\right)=\frac{\exp \left(-E_{\mathrm{pot}} / k T\right)}{\int \underset{\substack{(n-1) \\-\infty}}{+\infty} \int \exp \left(-E_{\mathrm{pot}} / k T\right) \mathrm{d} \Phi_{2} \ldots \mathrm{d} \Phi_{n}} ;
$$

dabei sind die Integrationsgrenzen passend abgeändert. Ferner ist angenommen, daß kein äußeres Feld wirkt. Der mittlere Winkel

$$
\varphi=\frac{1}{n} \sum_{v=1}^{n} \Phi_{v}
$$

sei vorgegeben; wir nennen $\varphi$ den. Winkel des Moleküls.

Zur Bestimmung des Wechselwirkungspotentials zweier Moleküle ändern wir nun die in 2. angestellten Betrachtungen wie folgt. Man denke sich zunächst ein Molekül eines Paares mit dem Winkel $\Psi=\Psi_{\mu}(\mu=1, \ldots, n)$ völlig in Ruhe, alle Winkel gegen die Ebene durch die beiden Achsen gemessen. Dann hat die $v$-te Gruppe des zweiten Moleküls mit dem mittleren Winkel $\varphi$ in bezug auf das erste das Potential

$$
{ }_{n-1}^{1} U\left(\Phi_{\nu}, \Psi\right)
$$

$U(\varphi, \psi)$ nach $(2,3)$. Mit der Verteilung $(5,2)$ ergibt sich dann als Mittelwert der Summe dieser Wechselwirkungspotentiale für alle Gruppen zusam. men

$$
\begin{aligned}
& \frac{1}{n-1} \sum_{\nu=1}^{n} \underset{\substack{(-\infty \\
-\infty}}{\stackrel{+\infty}{+\infty}} \int w\left(\Phi_{1}, \ldots, \Phi_{n}\right) \cdot U\left(\Phi_{\nu}, \Psi\right) \mathrm{d} \Phi_{2} \ldots \mathrm{d} \Phi_{n} \\
& \quad \approx(n-1)\left\{a_{0}+a_{1} \cos 2 \Psi+\left(a_{1}+a_{11} \cos 2 \Psi\right) \cos 2 \varphi \frac{\sqrt{\pi}}{2} \cdot \exp \left[-\frac{k T}{9 A}\left(n-\begin{array}{c}
1 \\
n
\end{array}\right)\right] \cdot \sqrt{k_{k T n} A} \cdot \Phi \sqrt{\frac{k T n}{3 A}}\right\},
\end{aligned}
$$

$\varphi$ laut $(5,3) ; \Phi$ bedeutet das Fehlerintegral. Das Integral erhält man durch die Substitution

$$
\delta_{v}=\Phi_{v}-\Phi_{v-1}, \quad v=2, \ldots, n
$$

nach einigen Rechnungen bei unwesentlichen Vernachlässigungen. $(5,4)$ vereinfacht sich erheblich, wenn man die Funktion

$$
f(x)=\frac{1}{x} \Phi(x) e^{-x^{2} / 2}, \quad x=\sqrt{k T n / 3 A},
$$

durch die Gerade $f(x)=1,3-0,68 x$ approximiert, was in dem in Frage kommenden $x$-Bereich von 0,35 bis 1,2 eine sehr gute Näherung ist (Abb. 8);

16 W. J. TAYlor, J. Chem. Phys. 16, 257 [1948]. diesen $x$-Bereich erhält man für $150<T<400$, $18<n<40, A \approx 10^{-12} \mathrm{erg}$. Der Amplitudenfaktor in $(5,4)$, welcher mit $M(T, n)$ bezeichnet sei, ist

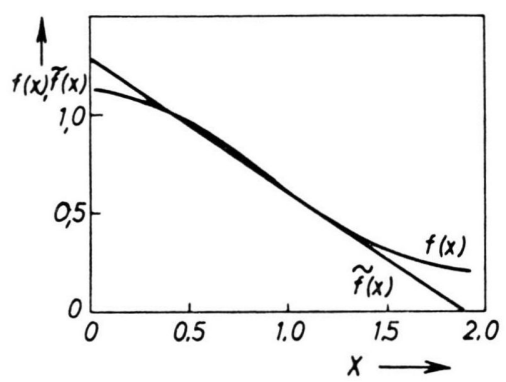

Abb. 8. Approximation von $f(x)$ durch $\tilde{f}(x)$. 
dann

$$
M(T, n)=1,15-0,61 \sqrt{k T n / 3 A},
$$

und das Potential wird

$$
\begin{aligned}
U(\varphi, \Psi) \cong & (n-1)\left[a_{0}+a_{1} \cos 2 \Psi\right. \\
& \left.+\left(a_{1}+a_{11} \cos 2 \Psi\right) \cos 2 \varphi M(T, n)\right] .
\end{aligned}
$$

Die Potentialschwelle wird also mit wachsendem $T n$ kleiner.

Um nun auch die Vibration des anderen Moleküls des Paares zu berücksichtigen, schreiben wir seinen Gruppen die Winkel $\Psi_{v}$ zu mit dem Mittelwert

$$
\psi=\frac{1}{n} \sum_{\nu=1}^{n} \Psi_{\nu}
$$

und rechnen dann genau so wie oben, indem das zweite Molekül nunmehr als starr mit dem Winkel $\varphi$ angesehen wird. Der dabei begangene, schwer übersehbare Fehler ist vermutlich nicht groß. Man erhält als mittleres Wechselwirkungspotential zwischen zwei vibrierenden Molekülen

$$
\begin{array}{r}
\overline{\bar{U}}(\varphi, \psi)=(n-1)\left[a_{0}+(\cos 2 \varphi+\cos 2 \psi) a_{1} M(T, n)\right. \\
\left.+\cos 2 \varphi \cos 2 \psi a_{11} M^{2}(T, n)\right] . \quad(5,6)
\end{array}
$$

Verglichen mit den Potentialformeln $(2,7)$ tritt also zu $a_{1}$ der Faktor $M$, zu $a_{11}$ der Faktor $M^{2}$ hinzu.

Es erhebt sich die Frage, wie sich die Ergebnisse von 4. ändern, wenn diese Faktoren berücksichtigt werden. Beeinflußt wird zunächst im monoklinen Gitter die Lösung der Gln. $(4,16 ; 4,17)$ und $(4,10$; $4,11)$. Nimmt man einmal an, $B_{1}$ und $B_{3}$ seien nicht sehr von den früheren Werten verschieden, so kann die Näherung $2 \pi r \approx 0,175 ; \delta \approx \pm \pi / 2$ von $(4,16$; $4,17)$ beibehalten werden. Geht man mit

$$
\cos \delta \approx 0,24 \cdot 10^{-14} \tau M\left(B_{3}-B_{1}\right)
$$

in die Gln. $(4,16 ; 4,17)$ ein, so ergibt sich nach einigen Umformungen und Entwicklungen nach kleinen Größen

$$
B_{1} \approx 4,5 \AA, \quad B_{3} \approx 4,6 \AA .
$$

Bei beiden Werten zeigt sich eine so schwache $\mathrm{Ab}$ hängigkeit von $n$ und $T$, daß sie unterhalb der einige Prozent betragenden Rechengenauigkeit liegt; sie erfüllen außerdem unsere Annahme. - Der hexagonale Achsenabstand $B$ folgt aus

$$
\frac{\mathrm{d} a_{0}}{\mathrm{~d} B}=-M^{2} \frac{\mathrm{d} a_{11}}{\mathrm{~d} B}
$$

zu $B=4,65+0,1 \cdot M^{2} \approx 4,71 \AA$; auch hier ist die Änderung mit $T$ und $n$ sehr gering, sie beträgt höchstens $1 / 4 \%$ in den betrachteten Intervallen.
Nunmehr läßt sich die neue Lage des Umwand. lungspunktes $T_{\mathrm{u}}$ durch Gleichsetzen von $(4,15)$ und $(4,21)$ untersuchen, nachdem auch dort die Faktoren $M$ und $M^{2}$ eingefügt sind. Man wird in guter Näherung auf die Gleichung

$$
\tau M^{2}=\text { const } \approx 10^{15}
$$

geführt. Diese Zahl ist das 3- bis 4-fache des mittleren Meßwertes; wir gehen jedoch den Ursachen dieser Diskrepanz, welche wohl zuerst in dem zu groben Ritzschen Ansatz für die hexagonale Phase zu suchen sind, nicht weiter nach [vgl. auch die Bemerkung zu $(4,22)]$. $(5,8)$ liefert die Gleichung

$$
T=\frac{n 100}{(C+0,036 n)^{2}}
$$

für die Kettenlängenabhängigkeit der Umwandlungstemperatur; $C$ ist darin eine Funktion der Konstanten in $(5,8)$. Mit $C=1,9$ erhält man für $n=27$ die gemessene Temperatur $T=326^{\circ} \mathrm{K}$. Für diesen Wert von $C$ ergibt sich die Kurve A für $T(n)$ (Abb. 9). Verglichen mit der Meßkurve B ist der Anstieg immer noch zu groß.

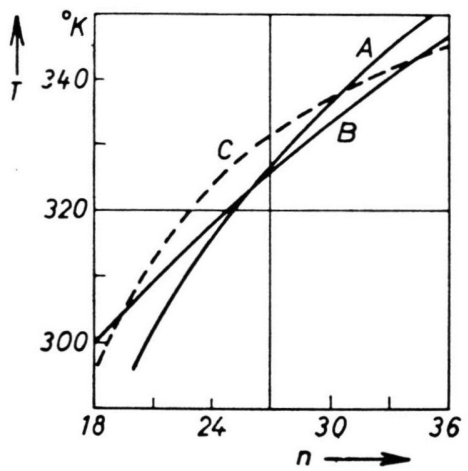

Abb. 9. Umwandlungstemperatur $T(n)$. B gemessen, A und C zwei Näherungen.

Eine bessere Auswertung der Gleichgewichtsbedingung liefert eine Abhängigkeit von der Gestalt

$$
n=27,8(10 \sqrt{\vartheta}-1,6-3,2 \vartheta), \quad T=n / \vartheta,
$$

dargestellt durch Kurve C; darin sind zwei Zahlenwerte gerade so bestimmt, daß die Kurve $\mathrm{C}$ in der Höhe von B liegt.

Allgemein ist bezüglich der Abweichungen der Ergebnisse von den experimentellen Messungen festzustellen, daß von vornherein einige für Paraffin sehr wenig bekannte Daten in die Rechnung hineingenommen werden mußten. Dadurch werden insbe- 
sondere gewisse Differenzen großer Zahlen sehr unsicher. Immerhin zeigt sich, daß sich auf dem beschrittenen Wege der Einbeziehung der Molekülverdrillung in die Theorie brauchbare Ergebnisse erzielen lassen.

Das Wechselwirkungspotential $(5,6)$ für vibrierende Moleküle ergibt auch eine andere Kettenlängenabhängigkeit der Umwandlungswärme (4,23). In $(4,24 ; 4,25)$ sind jetzt ebenfalls die Faktoren $M$ und $M^{2}$ einzuführen. Mit den Achsabständen $(5,7)$ und $B_{\text {hex }} \approx 4,71 \AA$ folgt dann mit einigen geringen Vernachlässigungen

$$
Q=\frac{n}{10}\left(2,46-\frac{3,2 n}{100}\right) \frac{\mathrm{kcal}}{\mathrm{Mol}}=n\left(1,7-\frac{2,2 n}{100}\right) \frac{10^{-14} \mathrm{erg}}{\text { Molekül }} .
$$

Die Umwandlungswärme $Q$ ist nicht für alle $\mathrm{Pa}$ raffine gemessen [Meßwerte und berechnete Funktion $Q(n)$ sind in Abb. 10 dargestellt]. Die vorliegenden Meßergebnisse ${ }^{17}$ stammen $z$. Tl. von verschiedenen Autoren, worauf die starke Streuung möglicherweise zurückzuführen ist; es sei unter diesem Vorbehalt dennoch darauf hingewiesen, daß die Umwandlungswärmen für geradzahlige Paraffine bis auf $\mathrm{C}_{28}$ größer als die der ungeradzahligen auszufallen scheinen. Diese Erscheinung könnte jedoch, falls sie wirklich existiert, von unserer Theorie ohnehin nicht erklärt werden.

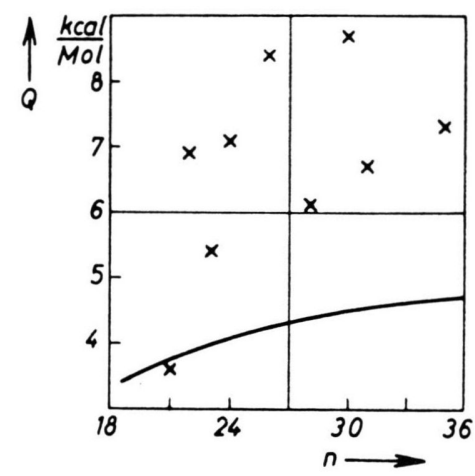

Abb. 10. Umwandlungswärme $Q$. Errechnete Kurve $(5,9)$ und Meßpunkte ${ }^{17}$.

Es sei betont, daß die obige Theorie keinerlei Anhaltspunkte für den Kettenlängenbereich bietet, in welchem eine Phasenumwandlung überhaupt auftritt; die durchgängige Beschränkung auf $18 \leqq n \leqq 36$ geschah lediglich auf Grund der physikalischen Be-

17 G. Timmermanns, Physico-Chemical Constants, Elsevier Publ. Comp., Amsterdam 1950. obachtungen. Zur Bestimmung des $n$-Bereichs müßte eine Theorie des Schmelzens von Paraffin entwickelt werden.

\section{An h ang}

Hier wird auf einige Ergänzungen der vorstehenden Theorie, die zur Durchführung derselben nicht unbedingt benötigt werden, und auf die Hofrmansche Theorie der Phasenumwandlung von Paraffinkristallen eingegangen.

\section{Das Rotationspotential eines starren Moleküls bei ruhenden Nachbarn}

MüLLER ${ }^{18}$ hat die potentielle Energie eines Moleküls im Kristallverband auf drei verschiedenen Wegen zu einigen fixen Winkelstellungen berechnet. Hier soll nun das Potential zu kontinuierlichem Winkel bestimmt werden. Ähnlich wie in 2. wird derjenige Weg gewählt, bei dem das Zentrum der van DER Walsschen Kräfte jeder $\mathrm{CH}_{2}$-Gruppe in den Schwerpunkt der positiven Ladungen mit dem Abstand $r=0,6 \AA$ von der Molekülachse angenommen ist. Das mittlere Molekül der Grundfigur (Abb. 1) sei drehbar und habe den Winkel $\varphi$ gegen die Seite $a$, alle übrigen haben den festen Winkel $30^{\circ}$. Das Wechselwirkungspotential zwischen zwei nunmehr isotrop vorausgesetzten Äthylengruppen verschiedener Moleküle gehorche der Beziehung ${ }^{14}$

$$
u=-2 \varepsilon\left(R_{\min } / R\right)^{6}+\varepsilon\left(R_{\min } / R\right)^{12} .
$$

$R$ bedeutet den Abstand der Ladungsschwerpunkte, $R_{\min }$ den Gleichgewichtsabstand und $\varepsilon$ die Energie des Paares im Gleichgewicht. Die Konstanten haben folgende Werte ${ }^{14}$ für

$$
\begin{array}{ll}
\text { Methan: } & R_{\min }=4,29 \AA, \quad \varepsilon \approx 19,5 \cdot 10^{-15} \mathrm{erg} ; \\
\text { Neon }: & R_{\min }=3,16 \AA, \quad \varepsilon \approx 5,16 \cdot 10^{-15} \mathrm{erg} .
\end{array}
$$

Diese Zahlen geben wegen der Ähnlichkeit der äußeren Elektronenschalen eine gewisse Orientierung über die entsprechenden Konstanten der Äthylgruppe [vgl. Bemerkung zu $(2,1)]$. $-\varepsilon$ und $R_{\min }$ werden sich aus der Sublimationswärme und dem Winkel minimaler potentieller Energie ergeben.

Die Gesamtenergie einer Gruppe soll sich wie in 2. additiv aus den Potentialen zwischen dieser und sämtlichen Gruppen der Nachbarmoleküle zusammensetzen. Die Moleküle seien unendlich lang. Wesentliche Beiträge werden nur von den sechs nächsten Nachbarn geliefert, der Rest ergibt einen vom Drehwinkel nahezu unabhängigen Beitrag. Im gewählten Modell ist der Mittelpunkt einer jeden C-C-Bindung Symmetriezentrum des Kristalls, so daß die Summe der Paarpotentiale nur für eine einzige Äthylengruppe ausgerechnet zu werden braucht. Das Potential des gesamten

18 A. Müller, Helv. Phys. Acta 9, 626 [1936]; Proc. Roy. Soc., Lond. A 127, 417 [1930]. 
Moleküls mit $n$ Gruppen ist dann im wesentlichen das $n$-fache. Es sind nach $(6,1)$ die reziproken 6. und 12 . Potenzen der Paarabstände $R$ für alle Paare aufzusummieren. Die Fourier-Entwicklungen der Summen sind $\sum R^{-6}=(38,8-0,39 \cos \varphi+0,20 \sin \varphi$

$$
+0,64 \cos 2 \varphi-1,07 \sin \varphi) 10^{-4} \AA^{-6},
$$

$\sum R^{-12}=(64,74-5,21 \cos \varphi+2,62 \sin \varphi$

$$
\begin{aligned}
& +8,45 \cos 2 \varphi-16,12 \sin 2 \varphi \\
& +0,48 \cos 3 \varphi+1,41 \sin 3 \varphi
\end{aligned}
$$

$-3,38 \cos 4 \varphi-2,22 \sin 4 \varphi+0,2 \cos 5 \varphi) 10^{-8} \AA^{-12}$.

Damit nun das Gesamtpotential

$$
U(\varphi)=-2 \varepsilon R_{\min }^{6} \sum R^{-6}+\varepsilon R_{\min }^{12} \sum R^{-12}
$$

für den Winkel $\varphi=30^{\circ}$ ein Minimum hat, muß dort $\mathrm{d} U / \mathrm{d} \varphi=0$ sein, woraus

$$
R_{\min }=3,83 \AA
$$

folgt. Dies paßt hinlänglich zu den Neon- und MethanWerten $(6,2)$ wie auch zu den Kristalldaten; der nächstmögliche Abstand zweier Gruppen eines der betrachteten Paare beträgt nämlich 3,65 A. Mit dem BunN schen Winkel ${ }^{13} \varphi_{\min }=48,8^{\circ}$ erhält $\operatorname{man} R_{\min }=2,98 \AA$. Bei dieser Überlegung ist natürlich vorausgesetzt, daß der gemessene mittlere Winkel mit dem Winkel minimaler potentieller Energie übereinstimmt, was bei niedrigen Temperaturen auch erfüllt ist.

$\varepsilon$ bestimmt sich aus der gemessenen Sublimationswärme $-1,62 \cdot 10^{-13} \mathrm{erg}$ pro Äthylengruppe ${ }^{14}$. Da eine Verteilungsfunktion $p(\varphi)$ für die Richtungen eines Moleküls nicht von vornherein bekannt ist, fordern wir

$$
\frac{1}{2} U\left(30^{\circ}\right)=-1,62 \cdot 10^{-13} \mathrm{erg} \text {. }
$$

Der Faktor $\frac{1}{2}$ tritt auf, weil die Energie des Paares gleichmäßig auf beide Äthylengruppen aufzuteilen ist. Es folgt

$$
\varepsilon=1,74 \cdot 10^{-14} \mathrm{erg} ;
$$

dieser Wert ist fast der gleiche wie der aus gaskinetischen Untersuchungen bestimmte $\varepsilon$-Wert des Methans.

Mit diesen Zahlen für $R_{\min }$ und $\varepsilon$ ergibt sich schließlich aus $(6,3)$ als Rotationspotential für die einzelne Athylengruppe

$$
\begin{aligned}
E(\varphi)=[ & -152-5,2 \cos \left(\varphi+26,5^{\circ}\right) \\
& +17,5 \cos 2\left(\varphi+32,4^{\circ}\right)+2,4 \cos 3\left(\varphi-26^{\circ}\right) \\
& \left.-6,9 \cos 4\left(\varphi-8,35^{\circ}\right)\right] \cdot 10^{-15} \mathrm{erg} ; \quad(6,4)
\end{aligned}
$$

die Rechengenauigkeit beträgt einige Prozent. Die maximale Potentialdifferenz ist $4,6 \cdot 10^{-14} \mathrm{erg} / \mathrm{CH}_{2}$-Gruppe; MüLler ${ }^{19}$ gibt für diese Differenz eine untere Schranke von $3 \cdot 10^{-14} \mathrm{erg}$ an.

Der Verlauf von $E(\varphi)$ (Abb. 11) zeigt nun allerdings ein zweites, wenig tieferes Minimum bei etwa $80^{\circ}$, was nicht in unser Modell paßt. Doch ist die Differenz zwischen den beiden Minimalwerten so klein

19 A. Müller, Proc. Roy. Soc., Lond. A 178, 227 [1941]. $\left(4 \cdot 10^{-15} \mathrm{erg}\right)$, daß sie durch die Rechenungenauigkeit bedingt sein könnte. Für spätere Rechnungen interessiert nur die $\pi$-periodische Komponente, so daß annähernd für ein Molekül mit $n$ C-Atomen gilt $E_{n}(\varphi) \approx(n-1)[-152+17,5 \cos 2 \psi] \cdot 10^{-15} \mathrm{erg} / \mathrm{Mol}$.

darin ist $n-1$ statt $n$ geschrieben, weil die Endgruppen nur etwa den halben Beitrag liefern.

\section{Bemerkungen zur Hoffmanschen Theorie der Phasenumwandlung}

Unter geringfügiger Modifikation des von Hoffman in seiner Arbeit über behinderte zwischenmolekulare Rotation im Paraffinkristall ${ }^{5}$ verwendeten Potentialansatzes kann man für das Paraffinmolekül auch ein $\tau$-periodisches, rechteckiges Topfpotential zugrunde legen, welches sich als Schematisierung des in Abb. 11 wiedergegebenen Verlaufs auffassen läßt. Die Tiefe des Topfes sei $U_{n}$, seine Breite $a . N_{0}$ sei die Anzahl der Moleküle mit Energien $U_{n}, N_{1}$ der Rest. Hoffman führt dann einen Fernordnungsparameter

$$
p=N_{0} /\left(N_{0}+N_{1}\right)
$$

ein und macht den BRAGG-Williamschen Ansatz

$$
U_{n}=p U_{0 n} \text {. }
$$

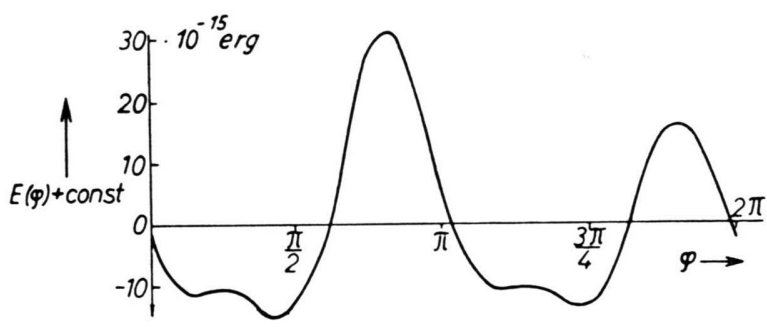

Abb. 11. Verlauf des Rotationspotentials $E(\varphi)+$ const $(6,4)$.

Aus dem Maxwell-Boltzmannschen Energieverteilungsgesetz folgt zunächst

$$
\frac{N_{1}}{N_{0}}=\frac{\pi-a}{a} e^{-U_{n} / k T}
$$

und weiter mit $\Omega=(\tau-a) / a$

$$
p / \ln \frac{\Omega p}{1-p}-\frac{k T}{U_{0 n}} \equiv y,
$$

woraus für $\Omega>e^{2}$ in gewissen Bereichen von $T$ eine dreiwertige Funktion $p(T)$ folgt (Abb. 12). Nach $(7,2)$ gilt

$$
p=\frac{1}{1+\Omega e^{-p U_{0 n} / k T}}>\frac{1}{1+\Omega},
$$

kleinere Werte kann $p$ für keine Temperatur annehmen. Diese Bedingung entsteht offensichtlich nur durch den Ansatz; man kann ihr keine physikalische Bedeutung beimessen, da es im hexagonalen Zustand kein 
stationäres $\pi$-periodisches Restpotential mehr geben kann und sich somit $p$ überhaupt nicht mehr definieren läßt. Die Beschränkung für $p$ stört hinfort jedoch nicht; man könnte sich ihrer durch eine Lineartransformation von $p$ entledigen.

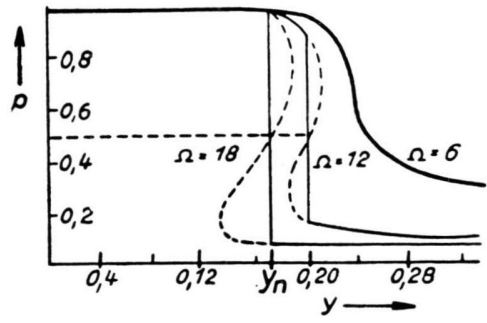

Abb. 12. Anteil $p$ der schwingenden Moleküle als Funktion der Temperatur; $y=k T / U_{0 n}$. Nach Hoffman.

Hoffman gibt als freie Energie pro Molekül

$F=\frac{U_{0 n}}{2}$

$$
-k T[(1-p) \ln \Omega-p \ln p-(1-p) \ln (1-\mathrm{p})]
$$

an. Als Umwandlungstemperatur $T_{\mathrm{u}}$ ist diejenige Temperatur definiert, für die diese Funktion $F(T)$ einen Doppelpunkt hat. Es schneiden sich die Äste für kleines und für großes $p$ (Abb. 13). Graphisch findet man für den Schnittpunkt

$$
y_{\mathrm{u}}=\frac{k T_{\mathrm{u}}}{U_{0 n}}=\frac{1}{2 \ln \Omega} .
$$

Analytisch kommt man ähnlich wie Hofrman auf folgende Weise zu diesem Ergebnis. Da der dreiwertige Teil von $p(y)$ (Abb. 12) einen physikalisch instabilen Zustand beschreibt, hat man ihn durch eine eindeutige

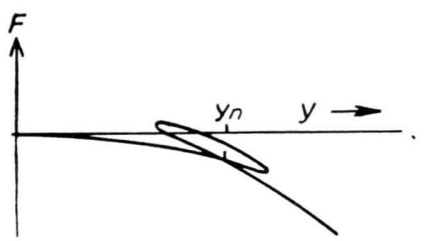

Abb. 13. Die freie Energie $F(T), \Omega=12$.

Funktion $p(y)$ zu ersetzen. Wir wählen eine Gerade $T=T_{\mathrm{u}}$ und postulieren damit einen Übergang erster Ordnung, welcher sich also nicht etwa zwangsläufig ergibt. Gl. $(7,2)$ gilt nicht mehr für $T_{\mathrm{u}}$, dagegen ist $p$ innerhalb gewisser Grenzen als unabhängige Variable anzusehen, nach der wir $F$ partiell differenzieren. Da $F$ minimal sein muß, verschwindet die erste Ableitung:

$$
\left(\frac{\partial F}{\partial p}\right)_{T_{\mathrm{u}}}=-U_{0 n} p+k T_{\mathrm{u}} \ln \frac{\Omega p}{1-p}=0 ;
$$

20 H. Fröhlich, Theory of Dielectrics, Clarendon Press, London 1949. das ist gerade $(7,2)$ mit drei Lösungen $p_{1}, p_{2}, p_{3}$ für $\Omega>e^{2} .(\partial F / \partial p)_{T_{1}}$ geht gegen $-\infty$ für $p \rightarrow 0$ und gegen $+\infty$ für $p \rightarrow 1$, also ist die mittlere Wurzel $p_{\text {. }}$ ein Maximum von $F\left(p, T_{\mathrm{u}}\right)$, die beiden anderen sind Minima. Nun sollen die beiden Minima gleich sein. Da

$$
\left(\frac{\partial^{2} F}{\partial p^{2}}\right)_{T_{\mathrm{u}}}=-U_{0 n}-\frac{k T_{\mathrm{u}}}{p(1-p)}
$$

symmetrisch in $p$ und $1-p$ ist, ist das genau dann der Fall, wenn das Maximum von $F$ bei $p=1 / 2$ liegt. Das liefert mit $(7,4)$ wieder $(7,3)$.

Für konstantes $\Omega$ ergibt sich auch hier Proportionalität von $n$ und $T_{\mathrm{u}}$; Hoffman begegnet dem durch Einbeziehung der Verdrillung nach einer empirischen Formel von F RöHLICH ${ }^{20}$.

\section{Die Energieniveaus behinderter Rotatoren und ihre Zustandssummen}

Man denke sich das starre Molekül im unveränderlichen Potentialfeld der Nachbarn um seine Achse drehbar. Das Potential $V_{n}(\varphi)$ soll wie in 7. für das monokline Gitter $\pi$-periodisch und topfförmig sein; der Topf habe die Tiefe $U_{n}$ und die Breite $a ; n$ ist wieder die Anzahl der C-Atome im Molekül. Für die SchröpingerGleichung

$$
\frac{\mathrm{d}^{2} u}{\mathrm{~d} \varphi^{2}}+\frac{2 I_{n}}{\hbar^{2}}\left[E-V_{n}(\varphi)\right] u=0
$$

mit der Periodizitätsbedingung $u(\varphi)=u(\varphi+2 \pi)$ erhält man die implizite Gleichung für die Eigenwerte ${ }^{21} E$ $\mathfrak{C O}_{\mathfrak{D}} \varkappa(\pi-a) \cos k a+\frac{\varkappa^{2}-k^{2}}{2 \varkappa k} \operatorname{Sin} \varkappa(\pi-a) \sin k a= \pm 1$;

darin ist $\hbar=h / 2 \pi$ das Plancksche Wirkungsquantum,

$$
I_{n}=n \cdot 1,027 \cdot 10^{-39} \mathrm{~cm}^{2} \mathrm{~g}
$$

das Trägheitsmoment des Moleküls um seine Längsachse und

$$
\varkappa^{2}=\frac{2 I_{n}}{\hbar^{2}}\left(U_{n}-E\right), \quad k^{2}=\frac{2 I_{n}}{\hbar^{2}} E .
$$

Bekannt sind die Eigenwerte im Grenzfall $a \rightarrow 0$, $U_{n} \cdot a \rightarrow$ const. Für den allgemeinen Fall ist Gl. $(8,2)$ zu analysieren. $E \leqq 0$ kann für keinen Eigenwert gelten, da dann $(8,1)$ keine beschränkte Lösung besitzt. Nach $(6,5)$ gilt größenordnungsmäßig $U_{n} \approx 2 n \cdot 10^{-14} \mathrm{erg}$; mit

$$
\frac{I_{n}}{\hbar^{2}}=n \cdot 0,925 \cdot 10^{15} \mathrm{erg}^{-1}
$$

ist dann für $E<U_{n}-\varepsilon, \varepsilon \lesssim \frac{1}{1000} \cdot U_{n}, \quad n \geqq 20$

$$
\varkappa^{2} \lesssim 4 n^{2} \cdot 10^{-2} \geqq 16 \text {. }
$$

Mit $a<\pi / 3$ wird $x(\pi-a) \gtrsim 8$ und

$$
\operatorname{Soj} x(\pi-a) \approx \operatorname{Sin} x(\pi-a) \approx \frac{1}{2} e^{\varkappa(x-a)} \text {. }
$$

21 S. FlügGe u. Marschald, Rechenmethoden der Quantenmechanik, Springer-Verlag, 2. Aufl., Berlin 1952. 
Beschränkt man sich zunächst auf Eigenwerte $0 \leqq E \leqq U_{n}$, so geht die Eigenwertbedingung $(8,2)$ über in

$$
\cos k a+\frac{\varkappa^{2}-k^{2}}{2 \varkappa k} \sin k a \lesssim \pm 2 e^{-\varkappa(x-a)} \approx 0 .
$$

Es ist zweckmäßig, $y^{2}=E / U_{n}$ und $C^{2}=2 I_{n} U_{n} / \hbar^{2}$ zu setzen; es gilt $0<y^{2}<1$ und $C^{2} \gtrsim 16000$. Gl. $(8,5)$ liefert

$$
\cot a C y \approx \frac{1}{2} \sqrt{\frac{1}{y^{2}\left(1-y^{2}\right)}-4 \cdot \operatorname{sign}\left(y^{2}-\frac{1}{2}\right) .}
$$

Die rechte Seite ist mit $y$ langsam veränderlich, die linke oszilliert schnell und hat Pole bei $a C y=m \pi$. Da die rechte Seite mit $y^{2}$ von $-\infty$ bis $+\infty$ monoton zunimmt, sind die Eigenwerte $E_{m}<U_{n}$

$$
E_{m}=\frac{\hbar^{2}}{2 I_{n}}\left[\frac{\left.\pi\left[(m+1) / 2-\varepsilon_{m}\right)\right]}{a}\right]^{2}, \quad m=1,2,3, \ldots,
$$

worin $\varepsilon_{m}$ zwischen 0 und 1 liegt und mit $m$ wächst. Aus $(8,5)$ folgt, daß die Eigenwerte paarweise dicht beieinander liegen und einfach sind. Für unsere Zwecke genügt es zu sagen, die Zahlen

$$
e_{m} \approx \frac{\hbar^{2}}{2 I_{n}}\left(\frac{\pi m}{a}\right)^{2}, \quad m=1,2,3, \ldots,
$$

seien zweifach entartete Eigenwerte.

Zur Untersuchung der Eigenwerte $E>U_{n}$ kann man

$$
\varkappa=i \sigma, \quad \sigma=\frac{2 I_{n}}{\hbar^{2}}\left(E-U_{n}\right)
$$

in $(8,2)$ einsetzen:

$$
\cos \sigma(\pi-a) \cos k a-\frac{\sigma^{2}-k^{2}}{2 \sigma k} \sin \sigma(\pi-a) \sin k a= \pm 1 \text {. }
$$

Mit $x \equiv E / U_{n}>1$ ist

$$
\frac{\sigma^{2}+k^{2}}{2 \sigma k}=\frac{2 x-1}{2 \sqrt{(x-1) x}}>1,
$$

insbesondere ist für $x \approx 1$

$$
\frac{\sigma^{2}+k^{2}}{2 \sigma k} \approx \frac{1}{2 \sqrt{x-1}} \gg 1
$$

In diesem Fall ist in

$$
\begin{gathered}
\cos \{(\pi-a) C \sqrt{x-1}\} \cos a C \sqrt{x} \\
= \pm 1+\frac{1}{2 \sqrt{x-1}} \sin \{(\pi-a) C \sqrt{x-1}\} \\
\cdot \sin \{a C \sqrt{x}\}
\end{gathered}
$$

das Argument $(\pi-a) C \sqrt{x-1}$ schnell veränderlich. Die Eigenwerte liegen, wieder in Paaren, dicht bei den Nullstellen $m \pi$ von $\sin \{(\pi-a) C \sqrt{x-1}\}$. Für die ersten Eigenwerte $E_{\mathrm{m}}{ }^{*}$ oberhalb $U_{n}$ gilt die Näherung $e_{m}{ }^{*}=U_{n}+\frac{\hbar^{2}}{2 I_{n}}\left(\frac{m \pi}{\pi-a}\right)^{2}, \quad m=1,2,3, \ldots$, klein $;(8,8)$

jede dieser Zahlen ist zweimal zu nehmen.

Für $x \gg 1$ wird $\sigma \approx k,\left(\sigma^{2}+k^{2}\right) / 2 \sigma k \approx 1$. Schreibt man $(8,7)$ in der Form

$$
\begin{gathered}
\cos C\{(\pi-a) \sqrt{x-1}+a \sqrt{x}\} \\
= \pm 1+\left\{\frac{\sigma^{2}+k^{2}}{2 \sigma k}-1\right\} \sin \{(\pi-a) C \sqrt{x-1}\} \\
\cdot \sin \{a C \sqrt{x}\}
\end{gathered}
$$

so sieht man, daß Paare von Eigenwerten bei

$$
(\pi-a) \sqrt{x-1}+a \sqrt{x}=\frac{m \pi}{C}
$$

auftreten. Wegen $\sqrt{x-1} \approx \sqrt{x}$ haben wir für große $x$ und $m$ die Näherung

$$
e_{m}^{*}=U_{n}+\frac{\hbar^{2}}{2 I_{n}} m^{2}, \quad m \text { groß; }
$$

diese Zahlen können wieder als zweifach entartete Eigenwerte $E_{m}>U_{n}$ angesehen werden. - Aus $(8,9)$ sieht man, daß die Eigenwerte für mittlere $x$ nicht mehr in Paaren geordnet sind; die Paare für kleine und für große $x$ gruppieren sich um.

Man kann sich die Frage vorlegen, ob bei der Phasenumwandlung des Paraffinkristalls Quanteneffekte von Einfluß sind. Teller und Weigert ${ }^{22}$ haben eine ähnliche theoretische Untersuchung für das Äthanmolekül durchgeführt und festgestellt, daß schon bei sehr niedrigen Temperaturen nur geringe Unterschiede zwischen dem GibBsschen Zustandsintegral und der quantentheoretischen Zustandssumme bestehen. Da für Phasengleichgewichte die freie Energie

$$
F=-k T \ln \frac{\sum}{h}
$$

bestimmend ist, ist die Zustandssumme

$$
\Sigma=\sum_{m} \exp \left(-E_{m} / k T\right)
$$

mit den oben berechneten Eigenwerten $(8,6 ; 8,8 ; 8,10)$ aufzustellen. Man erkennt jedoch, daß die Energieniveaus für $a \gtrsim \pi / 10, n \gtrsim 20$ verglichen mit $k T_{300^{\circ}}$ $=4,14 \cdot 10^{-14} \mathrm{erg}$ so dicht liegen, daß die Zustands. summe auch hier durch das Integral ersetzt werden kann. Wegen des größeren Trägheitsmomentes und der höheren Temperaturen ist also in unserem Fall noch mehr als beim Äthan die klassische Behandlung, wie sie in 3., 4. angewendet ist, gerechtfertigt.

\section{Funktionalgleichungen für $p_{i}(\varphi, \psi)$ zum Varia- tionsproblem der freien Energie $F$}

Für die Verteilungsfunktionen $p_{i}(\varphi, \psi)$ aus 3. lassen sich durch Variation der freien Energie $(4,1)$ Funk tionalgleichungen gewinnen. Die Verteilungsfunktionen sollen den Integralbeziehungen $(3,1)$ und den Nebenbedingungen für die monokline Phase (siehe 4.) genügen. Wegen

$$
p_{1}\left(\varphi_{1}, \varphi_{3}\right)=p_{2}\left(-\varphi_{3},-\varphi_{1}\right)
$$

kann $p_{2}$ überall durch $p_{1}$ ausgedrückt werden; man braucht nur noch $p_{1}$ und $p_{3}$ zu variieren. Wir setzen

22 E. Teller u. K. Weigert, Nachr. Wiss. Göttingen, Math.phys. Kl. Fachgr. 2, Nr. 40, 218 [1933]. 
voraus, daß die vorliegende Variationsaufgabe eine Lösung besitzt: $\pi_{1}(\varphi, \psi)$ und $\pi_{3}(\varphi, \psi)$ sei ein Extremalenpaar,

$$
p_{i}(\varphi, \psi)=\pi_{i}(\varphi, \psi)+\varepsilon \eta_{i}(\varphi, \psi) \geqq 0, \quad i=1,3,
$$

seien Vergleichsfunktionen, welche sämtliche Nebenbedingungen erfüllen. Mit $F$ hat dann nach der üblichen Schlußweise auch diejenige Funktion ein Extremum, welche aus $F$ durch Addition der mit Lagrange- schen Faktoren bzw. Funktionen $\lambda_{i}$ multiplizierten $\mathrm{Ne}$ benbedingungen entsteht:

$$
\begin{aligned}
\hat{F}=F+\int_{0}^{2 \pi} \int & \lambda_{1}\left[p_{1}(\varphi, \psi)-1\right]+\lambda_{2}(\varphi, \psi)\left[p_{3}(\varphi, \psi)\right. \\
- & \left.p_{3}(\psi, \varphi)\right]+\lambda_{3}(\varphi)\left[p_{1}(\varphi, \psi)-p_{1}(\psi,-\varphi)\right] \\
& \left.+\lambda_{4}(\varphi)\left[p_{1}(\varphi, \psi)-p_{3}(-\varphi, \psi)\right]\right\} \mathrm{d} \varphi \mathrm{d} \psi
\end{aligned}
$$

Es gilt notwendig für $\varepsilon=0$ :

$$
\begin{array}{r}
\frac{\mathrm{d} F}{\mathrm{~d} \varepsilon}=\frac{\mathrm{d} \hat{F}}{\mathrm{~d} \varepsilon}=\int_{0}^{2 \pi}\left\{\eta_{1}(\varphi, \psi)\left[\frac{2}{k T} U_{1}(\varphi, \psi)-5 \ln \int_{0}^{2 \pi} \pi_{1}(\varphi, \chi) \mathrm{d} \chi+2 \ln \pi_{\mathbf{1}}(\varphi, \psi)-3+\lambda_{1}\right]\right. \\
+\eta_{3}(\varphi, \psi)\left[\frac{1}{k T} U_{3}(\varphi, \psi)+\ln \pi_{3}(\varphi, \psi)+1\right]+\lambda_{2}(\varphi, \psi)\left[\eta_{3}(\varphi, \psi)-\eta_{3}(\psi, \varphi)\right]+\lambda_{3}(\varphi)\left[\eta_{1}(\varphi, \psi)-\eta_{1}(\psi,-\varphi)\right] \\
\left.+\lambda_{4}(\varphi)\left[\eta_{1}(\varphi, \psi)-\eta_{3}(-\varphi, \psi)\right]\right\} \mathrm{d} \varphi \mathrm{d} \psi=0
\end{array}
$$

oder

$$
\begin{array}{r}
0=\int_{0}^{2 \pi} \int_{1}\left\{\eta_{1}(\varphi, \psi)\left[\frac{2}{k T} U_{1}(\varphi, \psi)-5 \ln \int_{0}^{2 \pi} \pi_{1}(\varphi, \chi) \mathrm{d} \chi+2 \ln \pi_{1}(\varphi, \psi)-3+\lambda_{1}+\lambda_{3}(\varphi)-\lambda_{3}(-\psi)+\lambda_{4}(\varphi)\right]\right. \\
\left.+\eta_{3}(\varphi, \psi)\left[\frac{1}{k T} U_{3}(\varphi, \psi)+\ln \pi_{3}(\varphi, \psi)+1+\lambda_{2}(\varphi, \psi)-\lambda_{2}(\psi, \varphi)-\lambda_{4}(-\varphi)\right]\right\} \mathrm{d} \varphi \mathrm{d} \psi
\end{array}
$$

Es muß nun gezeigt werden, daß die Lagrangeschen Multiplikatoren $\lambda_{i}$ stets so bestimmt werden können, daß die beiden eckigen Klammern, welche mit $K_{1}$ und $K_{3}$ bezeichnet seien, Elemente der Funktionenklassen der $\eta_{1}$ bzw. $\eta_{3}$ werden. Diese Klassen sind gekennzeichnet durch:

$$
\begin{gathered}
\eta_{\mathbf{3}}(\varphi, \psi)=\eta_{\mathbf{3}}(\psi, \varphi), \\
\int_{0}^{2 \pi} \int_{0} \eta_{i}(\varphi, \psi) \mathrm{d} \varphi \mathrm{d} \psi=0, \quad i=1,3, \\
\int_{0}^{2 \pi} \eta_{1}(\varphi, \psi) \mathrm{d} \psi=\int_{0}^{2 \pi} \eta_{1}(\psi,-\varphi) \mathrm{d} \psi, \\
\int_{0}^{2 \pi} \eta_{1}(\varphi, \psi) \mathrm{d} \psi=\int_{0}^{2 \pi} \eta_{3}(-\varphi, \psi) \mathrm{d} \psi, \\
\eta_{i}(\varphi+\pi, \psi)=\eta_{i}(\varphi, \psi+\pi)=\eta_{i}(\varphi, \psi) .
\end{gathered}
$$

Gl. $(9,2)$ liefert unter Ausnutzung der Symmetrie von $U_{i}(\varphi, \psi)$ [siehe $\left.(2,7)\right]$ und von $\pi_{3}(\varphi, \psi)$

$$
\lambda_{2}(\varphi, \psi)-\lambda_{2}(\psi, \varphi)=\frac{1}{2}\left[\lambda_{4}(-\varphi)-\lambda_{4}(-\psi)\right],
$$

also $\quad K_{3}(\varphi, \psi)=\frac{1}{k T} U_{3}(\varphi, \psi)+\ln \pi_{3}(\varphi, \psi)+1$

$$
-\frac{1}{2}\left[\lambda_{4}(-\varphi)+\lambda_{4}(-\psi)\right] \text {. }
$$

Aus $(9,6)$ folgt die $\pi$-Periodizität von $\lambda_{4}(\varphi)$ und $\lambda_{3}(\varphi)$. Die Gln. (9,3) legen $\lambda_{1}$ und $\int \lambda_{4}(\varphi) \mathrm{d} \varphi$ fest. $\lambda_{3}(\varphi)$ ist nur bis auf eine additive Konstante bestimmt; man kann $\int \lambda_{3}(\varphi) \mathrm{d} \varphi=0$ wählen. Schließlich folgen aus $(9,4 ; 9,5)$ zwei lineare inhomogene Gleichungen mit nichtverschwindender Determinante für $\lambda_{3}(\varphi)$ und $\lambda_{4}(\varphi)$. Damit ist gezeigt, daß sich die $\lambda_{i}$ zu jedem Extremalenpaar $\pi_{1}, \pi_{3}$ so bestimmen lassen, daß die beiden eckigen Klammern $K_{1}, K_{3}$ in $(9,1)$ den Funktionenklassen der $\eta_{1}, \eta_{3}$ angehören.

Nimmt man nun $K_{1}, K_{3} \neq 0$ an, so braucht man nur $\eta_{i} \sim K_{i}$ zu wählen, um in Widerspruch zu $(9,1)$ zu kommen. Daher gelten folgende notwendige Bedingungen für Extremalen $p_{1}, p_{3}$ :

$$
\begin{aligned}
& \frac{U_{1}(\varphi, \psi)}{k T}-\frac{5}{2} \ln \int p_{1}(\varphi \cdot \chi) \mathrm{d} \chi+\ln p_{1}(\varphi, \psi) \\
& \quad+\frac{1}{2}\left[\lambda_{1}+\lambda_{3}(\varphi)-\lambda_{3}(-\psi)+\lambda_{4}(\varphi)\right]=0,
\end{aligned}
$$

$\frac{U_{3}(\varphi, \psi)}{k T}+\ln p_{3}(\varphi, \psi)-\frac{1}{2}\left[\lambda_{4}(-\varphi)+\lambda_{4}(-\psi)\right]=0$.

Man entnimmt ihnen zum Beispiel, daß $p_{i}$ die Gestalt $p_{i}(\varphi, \psi)=f_{i 1}(\varphi) f_{i 2}(\psi) \exp \left[U_{i}(\varphi, \psi) / k T\right]$

hat. Es mag hier darauf hingewiesen werden, daß in der Fernordnungstheorie ähnlicher Probleme häufig der vereinfachte Ansatz

$$
p_{i}(\varphi, \psi) \sim \exp \left[U_{i}(\varphi, \psi) / k T\right]
$$

gemacht wird.

Geht man mit $(9,7)$ in die freie Energie $(4,1)$ ein, so erhält man als Extremalwert

$$
\begin{aligned}
\frac{F}{k T}=- & \lambda_{1}=\tau\left(2 a_{0}^{(1)}+a_{0}^{(3)}\right)-\frac{5}{2 \pi} \int \ln x_{1}(\varphi) \mathrm{d} \varphi \\
& +\frac{1}{4 \pi^{2}} \iint\left\{2 \ln p_{1}(\varphi, \psi)+\ln p_{3}(\varphi, \psi)\right\} \mathrm{d} \varphi \mathrm{d} \psi,
\end{aligned}
$$

in Übereinstimmung mit $(4,12)(\tau=n / k T)$. 


\section{Weitere kombinatorische Ausdrücke für die Entropie}

In 3. bestimmten wir Korrekturfaktoren $\Gamma_{1}, \Gamma_{3}$ $(3,5)$ für die Kombinationszahl $G(3,3) . \Gamma$ sollte der Quotient sein aus der Zahl tatsächlich möglicher Kombinationen $(X)_{L} /(P)_{L}$ und der Gesamtzahl aller bis dahin gezählten Kombinationen; dafür wurde $L ! /(X)_{L}$ gewählt, während doch $G$ nur gleich $(X)_{L} /(P)_{L}$ war. Wir bestimmen deshalb ein $\varepsilon_{1}<1$ so, da

$$
\frac{\left(X_{1}\right)_{L}}{\left(P_{2}\right)_{L}}=\left(\frac{L !}{\left(X_{2}\right)_{L}}\right)^{\varepsilon_{1}},
$$

also, unter Benutzung von Symmetrierelationen,

$$
\varepsilon_{1}=\frac{\iint p_{1}(\varphi, \psi) \ln p_{1}(\varphi, \psi) \mathrm{d} \varphi \mathrm{d} \psi}{\int x(\varphi) \ln x(\varphi) \mathrm{d} \varphi}-1 .
$$

Andererseits ist aber auch die Zahl der tatsächlich möglichen Kombinationen im Zähler von $\Gamma$ mit $(X)_{L} /(P)_{L}$ zu hoch beziffert, da ja nur die Konfiguration eines Nachbarmoleküls berücksichtigt ist. Wir potenzieren deshalb auch diese Zahl mit einer Zahl kleiner als 1 und wählen sie gleich $\varepsilon_{1}$. Das liefert statt $\Gamma_{1}$ den Korrekturfaktor

$$
\hat{\Gamma}_{\mathbf{1}}=\left[\frac{\left(X_{1}\right)_{L}}{\left(P_{1}\right)_{L}} / \frac{L !}{\left(X_{2}\right)_{L}}\right]^{\varepsilon_{1}}
$$

und als vorläufige Kombinationszahl

$$
G \hat{\Gamma}_{1}=\left[\left(X_{1}\right)_{L} /\left(P_{1}\right)_{L}\right]^{\varepsilon_{1}}
$$

Dies ist noch durch die zweite Korrekturwahrscheinlichkeit $\Gamma_{3}$ abzuändern, als welche wir analog

$$
\left[\frac{\left(X_{2}\right)_{L}}{\left(P_{3}\right)_{L}} / \frac{L !}{\left(X_{2}\right)_{L}}\right]^{\varepsilon_{2}}
$$

wählen mit

$$
\left[\frac{L !}{\left(X_{2}\right)_{L}}\right]^{\varepsilon_{2}}=\left[\begin{array}{l}
\left(X_{1}\right)_{L} \\
\left(P_{1}\right)_{L}
\end{array}\right]^{\varepsilon_{1}}=\left[\begin{array}{c}
L ! \\
\left(X_{2}\right)_{L}
\end{array}\right]^{\varepsilon_{1}^{2}} ;
$$

es ist also $\varepsilon_{2}=\varepsilon_{1}{ }^{2}$. Die Gesamtzahl aller Anordnungen wird nun

$$
G_{L}=\left[\left(X_{2}\right)_{L} /\left(P_{3}\right)_{L}\right]^{\varepsilon_{1}{ }^{2}},
$$

und mit $(10,1)$ ergibt dies den neuen Entropieausdruck analog $(3,7)$

$$
\begin{aligned}
& \underset{k}{S}=\left[\frac{\iint p_{1} \ln p_{1} \mathrm{~d} \varphi \mathrm{d} \varphi}{\int x \ln x \mathrm{~d} \varphi}-1\right]^{2}\left(\int x \ln x \mathrm{~d} \varphi\right. \\
& \left.-\iint p_{3} \ln p_{3} \mathrm{~d} \varphi \mathrm{d} \psi\right) .
\end{aligned}
$$

Mit Benutzung der Verteilungsfunktion $w(\varphi, \psi, \chi)$ für Dreiecke läßt sich folgendermaßen ein Entropieausdruck herleiten: es gibt $\left(P_{3}\right)_{L} /(W)_{L}$ Möglichkeiten, an das Paar $(4,1)$ das Dreieck $(1,3,4)$ in der richtigen Verteilung anzufügen, ohne auf die Konfiguration des Dreiecks $(1,2,3)$ zu achten; es ist

$$
(W)_{L}=\prod_{i, j, l}\left[w\left(k_{i}, k_{j}, k_{l}\right) L\right] ! .
$$

Dem Fehler trägt der folgendermaßen gewonnene Korrekturfaktor $\Gamma$ Rechnung: Paar 1 kann, wenn nur die Konfiguration von Molekül 1 berücksichtigt wird, auf $(X)_{L} /\left(P_{1}\right)_{L}$ Arten angefügt werden. Unter diesen gibt es $\left(P_{1}\right)_{L} /(W)_{L}$ Anordnungen, die die Konfiguration des Paares 2 berücksichtigen, so da $\beta$

$$
\Gamma=\frac{\left(P_{1}\right)_{L}}{(W)_{L}} / \frac{(X)_{L}}{\left(P_{1}\right)_{L}}
$$

gesetzt werden kann. Die Gesamtzahl der Anordnungen wird ${ }^{6}$

$$
G_{L}=\frac{\left(P_{1}\right)^{\frac{2}{L}}\left(P_{3}\right)_{L}}{(W)^{2}(X)_{L}}
$$

und die Entropie

$$
\begin{aligned}
\frac{S}{k}=\iint\left\{2 p_{1} \ln p_{1}+p_{3} \ln p_{3}\right\} \mathrm{d} \varphi \mathrm{d} \psi \\
-\iiint w \ln w \mathrm{~d} \varphi \mathrm{d} \psi \mathrm{d} \chi-\int x \ln x \mathrm{~d} \varphi .
\end{aligned}
$$

Durch Variation der freien Energie kann man daraus die Integralgleichung für $p_{1}(\varphi, \omega)$ gewinnen

$\exp \left[\frac{\lambda}{k T}+\frac{1}{k T} U_{3}(\psi, \omega)\right]$
$=\int_{0}^{2 \pi} \exp \left\{-\frac{1}{k T}\left[U_{1}(\varphi, \omega)+U_{2}(\varphi, \psi)\right]_{x_{1}(\varphi)}^{p^{2}(\varphi, \omega)}\right\} \mathrm{d} \varphi$,

$\lambda=$ const ist LaGRANGescher Multiplikator.

Integralgleichungen ähnlicher Art werden von $\mathrm{O}_{\mathrm{GuCHI}}$ und TAKaGI ${ }^{7}$ unter der Voraussetzung spezieller Annahmen für den Ising-Magneten angegeben.

\section{Vergleich eines exakten Entropieintegrals mit dem benutzten Näherungswert}

Eine Abschätzung des durch die Ansätze $(4,13)$ für die Logarithmen in den Integralen der Gln. $(4,3)$ bis $(4,9)$ entstehenden Fehlers ist im allgemeinen nicht leicht möglich. Im hexagonalen Fall jedoch lassen sich für den einfachen Ansatz

$$
p(\varphi, \psi)=\frac{1}{4 \pi^{2}}+b \cos 2(\varphi+\gamma) \cos 2(\psi+\gamma)
$$

für die Verteilungsfunktionen der Paarkonfigurationen, in dem $b$ und $\gamma$ Parameter sind, die folgenden Integrale exakt ausrechnen:

$\int_{0}^{2} \int_{0}^{\pi} \frac{\partial p}{\partial b} \ln p \mathrm{~d} \varphi \mathrm{d} \psi=-\frac{2}{\pi b}\left(E\left(4 \pi^{2} b\right)-\frac{\pi}{2}\right) \approx 4 \pi^{4} b$,

$\int_{0}^{2 \pi} \int \ln p \mathrm{~d} \varphi \mathrm{d} \psi=-4 \pi^{2}[2 \ln 2 \pi$

$$
\left.+\frac{1}{2} \sum_{n=1}^{\infty} \frac{1}{n}\left(\pi^{2} b\right)^{2 n}\left(\begin{array}{c}
2 n \\
n
\end{array}\right)^{2}\right]
$$

$E(k)$ bedeutet das vollständige elliptische Integral zweiter Gattung ${ }^{23}$; die Reihe in der zweiten Formel ist damit eng verwandt. Sie ist konvergent für $\left|4 \pi^{2} b\right| \leq 1$, ihr erstes Glied ist $2 \pi^{4} b$. 
Im Vergleich dazu ergeben die mit den Ansätzen $(4,13)$ berechneten Näherungsintegrale

$$
\begin{gathered}
\int_{0}^{2 \pi} \frac{\partial p}{\partial b} \ln p \mathrm{~d} \varphi \mathrm{d} \psi \approx 4 \pi^{4} b \\
\int_{0}^{2 \pi} \ln p \mathrm{~d} \varphi \mathrm{d} \psi \approx-4 \pi^{2}\left(2 \ln 2 \pi+2 \pi^{4} b^{2}\right) .
\end{gathered}
$$

Dies sind - wie nicht anders zu erwarten - die ersten Glieder der Reihenentwicklungen von $(11,1)$; sie stellen wegen der guten Konvergenz bei nicht zu großem $b$ eine brauchbare Näherung dar.
Für die Anregung und Fertigstellung dieser Arbeit sowie manche Hinweise ist der Verfasser Herrn Professor Dr. G. Schubert zu besonderem Dank verpflichtet. Für weitere Fragestellungen und wertvolle Diskussionen dankt er Herrn Professor Dr. H. A. Stuart. Die Durchführung der Arbeit wurde von der D e u t s che n Forschungsgemeinschaft in dankenswerter Weise unterstützt.

23 siehe etwa JAHnke-Emde, Tafeln höherer Funktionen, Leipzig 1948.

\title{
Die größtmögliche Ausbeute einer Ra+Be-Neutronenquelle
}

\author{
Von F. Berthold und P. Jensen * \\ Aus dem Max-Planck-Institut für Chemie, Mainz \\ (Z. Naturforschg. 13 a, 143-147 [1958]; eingegangen am 5. Dezember 1957)
}

\begin{abstract}
Es wurden $\mathrm{Rn}+\mathrm{Be}-\mathrm{Neutronenquellen} \mathrm{hergestellt,} \mathrm{bei} \mathrm{denen} \mathrm{ausgefrorenes} \mathrm{Radon} \mathrm{einer} \mathrm{Beryllium.}$ scheibe unter genau definiertem Raumwinkel gegenüberstand. Kontrollmessungen zeigten u. a., daß die $\alpha$-Teilchen nicht im Präparat vorgebremst wurden und daß kaum Rückstoßkerne die Ausfrierstelle verließen. Nach Umrechnung von $\mathrm{Rn}+\mathrm{Be}$ - auf $\mathrm{Ra}+\mathrm{Be}$-Quellen ergab sich die größtmögliche Ausbeute einer Ra+Be-Quelle zu 16800 Neutronen (ohne Photoneutronen) pro sec und mC Ra mit Folgeprodukten außer Polonium ${ }^{1}$.

Im Anhang wird gezeigt, daß als Hauptursache für die Verschiedenheit der Neutronenspektren von $\mathrm{Ra}+\mathrm{Be}$-Quellen die unterschiedliche Photoneutronenausbeute anzusehen ist.
\end{abstract}

Die Neutronenausbeute einer Ra + Be-Quelle ist deshalb nie optimal, weil die $\alpha$-Teilchen schon in der Radiumverbindung vorgebremst werden.

Nun wäre es interessant zu wissen - etwa zur Beurteilung der Güte von Neutronenquellen - , wo die obere Grenze der pro mC Ra erreichbaren Neutronenausbeute liegt.

Deshalb wurde eine $\mathrm{Rn}+\mathrm{Be}$-Quelle hergestellt, in der die $\alpha$-Teilchen wegen der hohen spezifischen Aktivität des Radons praktisch nicht vorgebremst werden. Damit wird es möglich, daß die Ausbeute einer $\mathrm{Rn}+\mathrm{Be}-$ Quelle ihren theoretischen Höchstwert erreicht. Durch eine Reihe von Kontrollmessungen wurde geprüft, ob die Bedingungen zur Erreichung dieses Höchstwertes tatsächlich gegeben sind. Um von der $\mathrm{Rn}+\mathrm{Be}$ - auf die $\mathrm{Ra}+\mathrm{Be}$-Quelle schließen zu können, muß man noch eine Korrektur anbringen für die Neutronen, die von den $\alpha$-Teilchen des Radiums (ohne Folgeprodukte) erzeugt werden.

\section{Die Rn + Be-Neutronenquelle (Abb. 1)}

Ein Glasrohr von etwa $14 \mathrm{~mm}$ Innendurchmesser, das mit flüssiger Luft gefüllt werden kann, ist auf

\footnotetext{
* verstorben am 17.8. 1955.
}

einen größeren Glaszylinder mit etwa $27 \mathrm{~mm} \phi$ aufgeschmolzen. Der letztere kann über einen (in Abb. 1 nicht gezeigten) Schliff $(29 \mathrm{~mm} \phi)$ mit einer Emanationsanlage in Verbindung gebracht werden. Die im $\mathrm{Ca}-\mathrm{Ofen}$ gut gereinigte Emanation wird an der durch die flüssige Luft gekühlten Stelle ausgefroren.

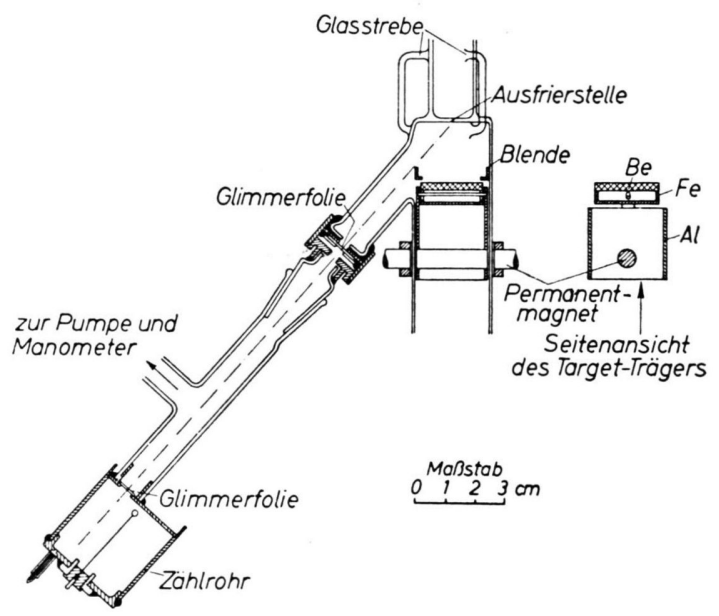

Abb. 1. Die Rn+Be-Neutronenquelle mit Zählrohr zur Messung der $a$-Reichweiten.

1 Vorläufige Mitteilung in Phys. Verh. 5, 3 [1954]. Wesent. liche Teile der vorliegenden Arbeit wurden als Diplomarbeit Freiburg 1955 ausgeführt. 